\title{
Idiopathic environmental intolerance attributed to electromagnetic fields (IEI-EMF): A systematic review of identifying criteria
}

\author{
Christos Baliatsas ${ }^{1,2^{*}}$, Irene Van Kamp ${ }^{2}$, Erik Lebret ${ }^{1,2}$ and G James Rubin ${ }^{3}$
}

\begin{abstract}
Background: Idiopathic environmental intolerance attributed to electromagnetic fields (IEI-EMF) remains a complex and unclear phenomenon, often characterized by the report of various, non-specific physical symptoms (NSPS) when an EMF source is present or perceived by the individual. The lack of validated criteria for defining and assessing IEI-EMF affects the quality of the relevant research, hindering not only the comparison or integration of study findings, but also the identification and management of patients by health care providers. The objective of this review was to evaluate and summarize the criteria that previous studies employed to identify IEI-EMF participants.

Methods: An extensive literature search was performed for studies published up to June 2011. We searched EMBASE, Medline, Psychinfo, Scopus and Web of Science. Additionally, citation analyses were performed for key papers, reference sections of relevant papers were searched, conference proceedings were examined and a literature database held by the Mobile Phones Research Unit of King's College London was reviewed.
\end{abstract}

Results: Sixty-three studies were included. "Hypersensitivity to EMF" was the most frequently used descriptive term. Despite heterogeneity, the criteria predominantly used to identify IEI-EMF individuals were: 1 . Self-report of being (hyper)sensitive to EMF. 2. Attribution of NSPS to at least one EMF source. 3. Absence of medical or psychiatric/ psychological disorder capable of accounting for these symptoms 4. Symptoms should occur soon (up to 24 hours) after the individual perceives an exposure source or exposed area. (Hyper)sensitivity to EMF was either generalized (attribution to various EMF sources) or source-specific. Experimental studies used a larger number of criteria than those of observational design and performed more frequently a medical examination or interview as prerequisite for inclusion.

Conclusions: Considerable heterogeneity exists in the criteria used by the researchers to identify IEI-EMF, due to explicit differences in their conceptual frameworks. Further work is required to produce consensus criteria not only for research purposes but also for use in clinical practice. This could be achieved by the development of an international protocol enabling a clearly defined case definition for IEI-EMF and a validated screening tool, with active involvement of medical practitioners.

\footnotetext{
* Correspondence: Christos.Baliatsas@rivm.nl

${ }^{1}$ Institute for Risk Assessment Sciences, Utrecht University, Utrecht, The

Netherlands

${ }^{2}$ National Institute for Public Health and the Environment (RIVM), Bilthoven,

The Netherlands

Full list of author information is available at the end of the article
} 


\section{Background}

Although the issue of idiopathic intolerances attributed to environmental exposures (IEI) first appeared in the scientific literature more than five decades ago [1], the possible underlying causes, as the term "idiopathic" suggests, remain unclear [2] and there is no widely accepted protocol for the identification of patients and treatment [3]. A representative example is the variety of physical symptoms without a clear pathological basis that are attributed by the patients to relatively low-level exposure to non-ionizing electromagnetic fields (EMF), emitted by sources such as mobile phone devices and base stations, high-voltage overhead powerlines, computer equipment and domestic appliances [4]. This phenomenon is better known within the public and scientific context as "electromagnetic hypersensitivity"(EHS), although since 2005 the term "Idiopathic Environmental Intolerance Attributed to EMF" (IEI-EMF) has been proposed by the World Health Organization (WHO) as an etiologically neutral description [5]. In this paper, the descriptive term "IEIEMF" is used.

According to the WHO [5], people with IEI-EMF are mainly characterized by the report of non-specific physical symptoms (NSPS), without a consistent pattern [6], such as redness, tingling, burning sensations in the facial area, fatigue, tiredness, lack of concentration, dizziness, nausea, heart palpitation and digestive disturbances. IEI-EMF is often accompanied by occupational, social and mental impairment $[4,7]$ and its estimated prevalence varies considerably, probably due to different methodological approaches; $1.5 \%$ in Sweden [6], 3.2\% in California [8], 3.5\% in Austria [9], 5\% in Switzerland [10] and 13.4\% in Taiwan [11]. Demographic characteristics such as age, gender and occupational status have repeatedly been associated with IEI-EMF $[6,10]$.

The experience and belief of IEI-EMF patients is in contrast with the scientific state of the art; results from systematic assessment of experimental and epidemiological evidence are consistent, concluding that a causal association of EMF exposure with symptomatic and other physiologic or cognitive reactions cannot be adequately supported [12-17]. IEI-EMF has been associated with psychological components [18-23] but their exact role is not clear. Although a possible effect of exposure cannot yet be ruled out because of methodological obstacles in research primarily regarding exposure assessment and study design $[14,16]$, more recent approaches stress the importance of looking into the interaction of environmental, biological, psychological and social pathways [24].

However, it is still controversial who should be categorised as having IEI-EMF. The lack of a validated, mutually accepted case definition and diagnostic instrument affects the quality of the research outcomes and increases the methodological heterogeneity, resulting in limited comparability between the studies. That stands in the way of a reliable estimation of the prevalence of IEI-EMF in the general population, proper meta-analysis of etiological evidence, the identification of health outcome patterns/profiles and contributes to a great deal of uncertainty regarding the characteristics, identification and management of this sensitivity by health care providers [25-27].

No systematic review has been performed yet focusing on the existing definitions and criteria for the identification of people with IEI-EMF. In light of the need to inform health care profesionals about relevant aspects of IEI-EMF and prepare the ground for discussion and consensus in the research community on widely supported case definition criteria, the present paper identified the relevant studies on IEI-EMF published to date, in order to summarize:

- The descriptive terms used to define IEI-EMF.

- The inclusion criteria and procedure for the identification of individuals with IEI-EMF.

\section{Methods}

\section{Search strategy for the identification of studies}

Initially, the following electronic databases were searched to detect relevant studies that were published from inception to April 2010: Embase (Elsevier B.V., Amsterdam, The Netherlands), Medline (US National Library of Medicine, Bethesda, Maryland), PsychInfo (American Psychological Association, Washington, DC). Web of Knowledge (Institute for Scientific Information, The Thomson Corporation, Stamford, Connecticut) and Scopus (Elsevier B.V., Amsterdam, The Netherlands). A wide range of (combined) keywords was used with regards to EMF exposure, sensitivity and related health outcomes, which is presented in Table 1. In addition to the electronic database searches, the reference sections of previous systematic reviews, key papers, international reports on EMF and health and research databases of websites focused on the issue of EMF such as the "EMF Portal" and the WHO webpage were checked for potentially

Table 1 Key search terms

\begin{tabular}{ll}
\hline Sensitivity: & Electrosensitivity, Electromagnetic hypersensitivity, \\
& Electrical sensitivity, Electromagnetic sensitivity, \\
& Electric hypersensitivity, IEI-EMF, Environmental \\
& intolerance, environmental illness. \\
\hline Exposure: & EMF, ELF, Electromagnetic field(s), Electromagnetic \\
& exposure, mobile telephones, mobile phone(s), \\
& Base stations, Powerlines, Celltowers, Antenna(e), \\
& UMTS, GSM, DECT, VDU, cell phones. \\
\hline Health Outcome: & Symptom(s), well-being, attributed symptoms, \\
& headache, fatigue. \\
\hline Time period & From inception - 2011. \\
\hline
\end{tabular}


Table 2 Experimental studies on IEI-EMF

\begin{tabular}{|c|c|c|c|c|c|c|c|}
\hline Reference & Study design & Recruitment & $\begin{array}{l}\text { Type of } \\
\text { sensitivity }\end{array}$ & $\begin{array}{l}\text { IEI-EMF sample } \\
\text { characteristics }\end{array}$ & $\begin{array}{l}\text { Identifying criteria } \\
\text { for IEI-EMF }\end{array}$ & $\begin{array}{l}\text { Main exclusion } \\
\text { criteria }\end{array}$ & $\begin{array}{l}\text { Identification/Case } \\
\text { definition methods } \\
\text { for IEl-EMF }\end{array}$ \\
\hline $\begin{array}{l}\text { Rea et al., } 1991 \\
\text { (USA) [28]. }\end{array}$ & Provocation & Voluntary participation. & General & $N=100$ & $\begin{array}{l}\text { Self-reported sensitivity } \\
\text { to EMF. }\end{array}$ & N.R/E. & $\begin{array}{l}\text { Subjective report, } \\
\text { medical examination. }\end{array}$ \\
\hline $\begin{array}{l}\text { Hamnerius } \\
\text { et al., 1993 } \\
\text { (Sweden) [29]. }\end{array}$ & Provocation & $\begin{array}{l}\text { IEI-EMF subjects } \\
\text { referred to a } \\
\text { health care service/ } \\
\text { institution. }\end{array}$ & VDU-specific & $N=30$ & $\begin{array}{l}\text { Report of "distinctive" } \\
\text { symptoms, occurrence } \\
\text { of the symptoms } \\
\text { within an hour from } \\
\text { being exposed } \\
\text { to VDU and } \\
\text { disappearance or } \\
\text { substantial reduction } \\
\text { within a few hours } \\
\text { after exposure } \\
\text { termination. }\end{array}$ & $\begin{array}{l}\text { Somatic or psychiatric } \\
\text { disorder that could } \\
\text { account for the } \\
\text { reported } \\
\text { symptomatology. }\end{array}$ & $\begin{array}{l}\text { Subjective report, } \\
\text { medical, psychiatric \& } \\
\text { psychological } \\
\text { examination. }\end{array}$ \\
\hline $\begin{array}{l}\text { Arnetz et al., } 1995 \\
\text { (Sweden) [30]. }\end{array}$ & Intervention & $\begin{array}{l}\text { IEI-EMF subjects } \\
\text { referred to a } \\
\text { health care service/ } \\
\text { institution. }\end{array}$ & General & $\begin{array}{l}N=20, m \cdot a=45 \\
f . g=75 \%\end{array}$ & $\begin{array}{l}\text { Report of symptoms } \\
\text { attributed to EMF } \\
\text { exposure. }\end{array}$ & $\begin{array}{l}\text { Somatic or psychiatric } \\
\text { disorder that could } \\
\text { account for the } \\
\text { reported } \\
\text { symptomatology. }\end{array}$ & $\begin{array}{l}\text { Subjective report, } \\
\text { medical \& psychiatric } \\
\text { examination. }\end{array}$ \\
\hline $\begin{array}{l}\text { Andersson } \\
\text { et al., } 1996 \\
\text { (Sweden) [31]. }\end{array}$ & Provocation & $\begin{array}{l}\text { IEI-EMF subjects } \\
\text { referred to a } \\
\text { health care service/ } \\
\text { institution. }\end{array}$ & General & $\begin{array}{l}N=17, m \cdot a=41.7 \\
f . g=70.6 \%\end{array}$ & $\begin{array}{l}\text { Experience of "typical" } \\
\text { symptoms of "electric } \\
\text { hypersensitivity" } \\
\text { when being in an } \\
\text { "electrical environment", } \\
\text { duration of at least } \\
6 \text { months, limitations } \\
\text { in daily functioning, } \\
\text { experience of } \\
\text { symptomatology within } \\
\text { less than } 30 \text { minutes } \\
\text { after exposure to electric } \\
\text { equipment under } \\
\text { experimental testing. }\end{array}$ & $\begin{array}{l}\text { Somatic or psychiatric } \\
\text { disorder that could } \\
\text { account for the } \\
\text { reported } \\
\text { symptomatology. }\end{array}$ & $\begin{array}{l}\text { Subjective report, } \\
\text { medical \& psychiatric } \\
\text { examination. }\end{array}$ \\
\hline $\begin{array}{l}\text { Bertoft et al., } 1996 \\
\text { (Sweden) [32]. }\end{array}$ & Provocation & $\begin{array}{l}\text { Voluntary participation } \\
\text { of IEI-EMF subjects. }\end{array}$ & General & $\begin{array}{l}N=5, \text { a.r }=46-60 \\
\text { f.g }=80 \%\end{array}$ & $\begin{array}{l}\text { Self-reported } \\
\text { hypersensitivity to EMF. }\end{array}$ & N.R/E. & Subjective report. \\
\hline $\begin{array}{l}\text { Toomingas, } 1996 \\
\text { (Sweden) [33]. }\end{array}$ & $\begin{array}{l}\text { Provocation } \\
\text { (case study) }\end{array}$ & $\begin{array}{l}\text { Voluntary participation } \\
\text { of a medical patient. }\end{array}$ & General & $\begin{array}{l}\mathrm{N}=1 \text { male } \\
\text { age }=35\end{array}$ & $\begin{array}{l}\text { Fear of a negative impact } \\
\text { of health caused by EMF } \\
\text { exposure, report of } \\
\text { symptoms attributed to EMF } \\
\text { such as fatigue, headache, } \\
\text { lack of concentration, } \\
\text { numbness \& paresthesia in } \\
\text { the arms, greasy feeling } \\
\text { in the palms, inability to } \\
\text { work due to the } \\
\text { reported symptoms. }\end{array}$ & N.R/E. & $\begin{array}{l}\text { Subjective report, } \\
\text { medical \& } \\
\text { neurological } \\
\text { examination. }\end{array}$ \\
\hline
\end{tabular}


Table 2 Experimental studies on IEI-EMF (Continued)

\begin{tabular}{|c|c|c|c|c|c|c|c|}
\hline $\begin{array}{l}\text { Sandström } \\
\text { et al., } 1997 \\
\text { (Sweden) [34]. }\end{array}$ & Provocation & $\begin{array}{l}\text { IEI-EMF subjects } \\
\text { treated to a } \\
\text { health care service/ } \\
\text { institution. }\end{array}$ & $\begin{array}{l}\text { VDT \& fluorescent } \\
\text { light-specific }\end{array}$ & $\begin{array}{l}N=10, m \cdot a=47 \\
f \cdot g=70 \%\end{array}$ & $\begin{array}{l}\text { Self-report of a } \\
\text { combination of } \\
\text { skin (mucous), eye \& } \\
\text { neurological } \\
\text { symptoms, attribution } \\
\text { of these symptoms to } \\
\text { EMF emitted from } \\
\text { VDT work, fluorescent } \\
\text { light or TV. }\end{array}$ & $\begin{array}{l}\text { Somatic or psychiatric } \\
\text { diseases severe enough } \\
\text { to require medical } \\
\text { treatment. }\end{array}$ & $\begin{array}{l}\text { Subjective report, } \\
\text { medical examination. }\end{array}$ \\
\hline $\begin{array}{l}\text { Hillert et al., } 1998 \\
\text { (Sweden) [35]. }\end{array}$ & Intervention & $\begin{array}{l}\text { IEl-EMF subjects } \\
\text { referred to a } \\
\text { health care service/ } \\
\text { institution. }\end{array}$ & General & $\begin{array}{l}N=10, m \cdot a=40 \\
f \cdot g=60 \%\end{array}$ & $\begin{array}{l}\text { Self-reported } \\
\text { hypersensitivity to } \\
\text { EMF, age between } \\
18-65 \text { y.o, being } \\
\text { employed for at least } \\
1 \text { week during the past } \\
3 \text { months, symptoms } \\
\text { had to show some } \\
\text { variation due to } \\
\text { perceived exposure } \\
\text { to EMF or proximity } \\
\text { to relative equipment. }\end{array}$ & $\begin{array}{l}\text { Medical or mental } \\
\text { disorder that could } \\
\text { account for the reported } \\
\text { symptomatology, long } \\
\text { period of sick leave, } \\
\text { unemployment. }\end{array}$ & $\begin{array}{l}\text { Subjective report, } \\
\text { medical examination. }\end{array}$ \\
\hline $\begin{array}{l}\text { Trimmel et al., } 1998 \\
\text { (Austria) [36]. }\end{array}$ & Provocation & Voluntary participation. & General & $N=36, a \cdot r=18-36$ & $\begin{array}{l}\text { Individual belief of an } \\
\text { "exceptional } \\
\text { reaction to EMF", a } \\
\text { score of }>50 \text { on } \\
\text { a continuous } \\
\text { rating scale } \\
\text { between } 0-100 \text {. }\end{array}$ & N.R/E. & Subjective report. \\
\hline $\begin{array}{l}\text { Flodin et al., } 2000 \\
\text { (Sweden) [37]. }\end{array}$ & Provocation & $\begin{array}{l}\text { IEI-EMF subjects } \\
\text { who were members } \\
\text { of a relative self-group } \\
\text { or registered to a } \\
\text { health care } \\
\text { service/institution. }\end{array}$ & General & $\begin{array}{l}N=15, m \cdot a=48.3 \\
f \cdot g=73.3\end{array}$ & $\begin{array}{l}\text { Attribution of } \\
\text { symptoms to } \\
\text { named EMF sources, } \\
\text { mean reaction should } \\
\text { occur within } 60 \text { minutes } \\
\text { of exposure, there } \\
\text { was no experience of } \\
\text { symptoms at home or } \\
\text { workplace when the } \\
\text { subject was considered } \\
\text { as "unexposed", } \\
\text { symptoms should } \\
\text { disappear within a } \\
\text { few days } \\
\text { after exposure. }\end{array}$ & $\begin{array}{l}\text { Slow reaction or denial } \\
\text { for participation } \\
\text { because of symptom } \\
\text { severity, undergoing } \\
\text { treatment for medical } \\
\text { conditions. }\end{array}$ & $\begin{array}{l}\text { Subjective report, } \\
\text { medical examination } \\
\text { (registered IEl-EMF } \\
\text { subjects). }\end{array}$ \\
\hline $\begin{array}{l}\text { Lohne-Rahm } \\
\text { et al., 2000 } \\
\text { (Sweden) [38]. }\end{array}$ & Provocation & $\begin{array}{l}\text { Voluntary participation } \\
\text { after description of the } \\
\text { study in newspaper } \\
\text { advertisements or IEI-EMF } \\
\text { subjects referred to a } \\
\text { health care service/ } \\
\text { institution. }\end{array}$ & General & $\mathrm{N}=12$ & $\begin{array}{l}\text { Report of skin } \\
\text { symptoms during } \\
\text { a } 30 \text {-minute exposure } \\
\text { to EMF \& symptom } \\
\text { duration of at } \\
\text { least } 6 \text { months. }\end{array}$ & $\begin{array}{l}\text { Diagnosed skin diseases, } \\
\text { slow, excessive or no } \\
\text { reactions during the } \\
\text { experiment. }\end{array}$ & $\begin{array}{l}\text { Subjective report, } \\
\text { medical examination } \\
\text { (referred IEl-EMF } \\
\text { subjects). }\end{array}$ \\
\hline
\end{tabular}


Table 2 Experimental studies on IEI-EMF (Continued)

\begin{tabular}{|c|c|c|c|c|c|c|c|}
\hline $\begin{array}{l}\text { Hillert et al., } 2001 \\
\text { (Sweden) [39]. }\end{array}$ & Intervention & $\begin{array}{l}\text { IEl-EMF subjects referred } \\
\text { to a health care service/ } \\
\text { institution. }\end{array}$ & General & $\begin{array}{l}N=16, m \cdot a=39.5 \\
f . g=81.3 \%\end{array}$ & $\begin{array}{l}\text { Self-reported } \\
\text { hypersensitivity to } \\
\text { electricity, experience } \\
\text { of change in symptoms } \\
\text { within } 24 \text { hours after } \\
\text { a perceived change in } \\
\text { exposure to EMF, a } \\
\text { history of VDU or } \\
\text { fluorescent lights as } \\
\text { the initial } \\
\text { triggering factors. }\end{array}$ & $\begin{array}{l}\text { Somatic or psychological } \\
\text { disorder that could } \\
\text { account for the } \\
\text { reported } \\
\text { symptomatology. }\end{array}$ & $\begin{array}{l}\text { Subjective report, } \\
\text { medical \& psychological } \\
\text { examination. }\end{array}$ \\
\hline $\begin{array}{l}\text { Lyskov et al., } 2001 \\
\text { (Sweden) [40]. }\end{array}$ & Provocation & $\begin{array}{l}\text { IEI-EMF subjects referred } \\
\text { to a health care service/ } \\
\text { institution. }\end{array}$ & General & $\begin{array}{l}N=20, m \cdot a=45.8 \\
f \cdot g=75 \% .\end{array}$ & $\begin{array}{l}\text { Self-reported } \\
\text { hypersensitivity } \\
\text { to EMF }\end{array}$ & N.R/E. & $\begin{array}{l}\text { Subjective report, } \\
\text { medical examination. }\end{array}$ \\
\hline $\begin{array}{l}\text { Hietanen } \\
\text { et al., 2002 } \\
\text { (Finland) [41]. }\end{array}$ & Provocation & Voluntary participation. & General & $\begin{array}{l}N=20, m \cdot a=49 \\
f \cdot g=65 \%\end{array}$ & $\begin{array}{l}\text { Self-reported } \\
\text { hypersensitivity to } \\
\text { EMF, experience of } \\
\text { symptoms during a } \\
\text { 30-minute } \\
\text { (provocation) } \\
\text { test period. }\end{array}$ & N.R/E. & $\begin{array}{l}\text { Subjective report, } \\
\text { medical examination. }\end{array}$ \\
\hline $\begin{array}{l}\text { Hillert et al., } 2002 \\
\text { (Sweden) [42]. }\end{array}$ & Intervention & $\begin{array}{l}\text { IEI-EMF subjects referred } \\
\text { to a health care service/ } \\
\text { institution. }\end{array}$ & General & $\begin{array}{l}N=22, m \cdot a=42 \\
f \cdot g=64 \%\end{array}$ & $\begin{array}{l}\text { Report of symptoms } \\
\text { assumed to be caused } \\
\text { by sensitivity to EMF. }\end{array}$ & $\begin{array}{l}\text { Medical or } \\
\text { psychological } \\
\text { condition that could } \\
\text { account for the } \\
\text { symptoms. }\end{array}$ & $\begin{array}{l}\text { Subjective report, } \\
\text { medical \& psychiatric } \\
\text { examination. }\end{array}$ \\
\hline $\begin{array}{l}\text { Mueller et al., } 2002 \\
\text { (Switzerland) [43]. }\end{array}$ & Provocation & Voluntary participation. & General & $\begin{array}{l}N=63, m \cdot a=49.5 \\
f \cdot g=51 \% .\end{array}$ & $\begin{array}{l}\text { Self-reported } \\
\text { sensitivity to EMF } \\
\text { or "Electrical } \\
\text { Hypersensitivity } \\
\text { Syndrome (EHS)". }\end{array}$ & N.R/E. & Subjective report. \\
\hline $\begin{array}{l}\text { Leitgeb et al., } 2003 \\
\text { (Austria) [44]. }\end{array}$ & Provocation & $\begin{array}{l}\text { Randomly selected } \\
\text { sample from } \\
\text { general population } \\
(\mathrm{N}=708) \text {. }\end{array}$ & General & a.r $=17-60$ & $\begin{array}{l}\text { Increased levels of } \\
\text { "electrosensibility", } \\
\text { defined as the individual } \\
\text { ability to perceive } \\
\text { electric or } \\
\text { electromagnetic } \\
\text { exposures without } \\
\text { necessarily } \\
\text { developing health } \\
\text { symptoms. }\end{array}$ & N.R/E. & $\begin{array}{l}\text { Measurement of } \\
\text { EMF perception } \\
\text { thresholds. }\end{array}$ \\
\hline $\begin{array}{l}\text { Österberg } \\
\text { et al., 2004 } \\
\text { (Sweden) [45]. }\end{array}$ & Provocation & $\begin{array}{l}\text { Randomly selected } \\
\text { sample from } \\
\text { general population } \\
(\mathrm{N}=13381) \text {, based on } \\
\text { Östergren et al. } \\
\text { (report) [46]. }\end{array}$ & General & $\begin{array}{l}N=16, m \cdot a=41.8 \\
f . g=50 \% .\end{array}$ & $\begin{array}{l}\text { Individual experience } \\
\text { the past } 2 \text { weeks of } \\
\text { "very much" physiologic } \\
\text { "annoyance" attributed } \\
\text { to FTL, and/or VDU } \\
\text { and/or other } \\
\text { electrical equipment. }\end{array}$ & $\begin{array}{l}\text { Report of long-term } \\
\text { sick leave, disability } \\
\text { pension, subjects } \\
\text { diagnosed with severe } \\
\text { medical condition that } \\
\text { required medication } \\
\text { (e.g. diabetes), } \\
\text { age of }>58 \text { y.o }\end{array}$ & $\begin{array}{l}\text { Subjective report, } \\
\text { medical examination. }\end{array}$ \\
\hline
\end{tabular}


Table 2 Experimental studies on IEI-EMF (Continued)

\begin{tabular}{|c|c|c|c|c|c|c|c|}
\hline $\begin{array}{l}\text { Belyaev et al., } 2005 \\
\text { (Sweden) [47]. }\end{array}$ & & & & $\begin{array}{l}N=7, m . a=44.8 \\
f . g=71.5 \%\end{array}$ & $\begin{array}{l}\text { Self-reported } \\
\text { hypersensitivity } \\
\text { to EMF. }\end{array}$ & $\begin{array}{l}\text { Smoking, regular } \\
\text { medication }\end{array}$ & \\
\hline $\begin{array}{l}\text { Frick et al., } 2005 \\
\text { (Germany) [48]. }\end{array}$ & Provocation & $\begin{array}{l}\text { Voluntary participation } \\
\text { after description } \\
\text { of the study in } \\
\text { a local newspaper. }\end{array}$ & General & $\begin{array}{l}N=30, m \cdot a=41.7 \\
f . g=77 \%\end{array}$ & $\begin{array}{l}\text { Self-reported } \\
\text { hypersensitivity to } \\
\text { named EMF sources, } \\
\text { attribution of severe } \\
\text { symptoms that limited } \\
\text { daily functioning \& } \\
\text { age between } \\
\text { 18-64 y.o. }\end{array}$ & $\begin{array}{l}\text { Not complainingor } \\
\text { not experiencing } \\
\text { limitations to daily } \\
\text { living due to the } \\
\text { reported } \\
\text { symptomatology. }\end{array}$ & Subjective report. \\
\hline $\begin{array}{l}\text { Wenzel et al., } 2005 \\
\text { (Germany) [49]. }\end{array}$ & Provocation & Voluntary participation. & $\begin{array}{l}\text { VDU \& } \\
\text { powerline- } \\
\text { specific }\end{array}$ & $\begin{array}{l}\mathrm{N}=3 \text { male } \\
\text { subjects, } \\
\text { m.a }=37 .\end{array}$ & $\begin{array}{l}\text { Concern about the } \\
\text { effects of EMF } \\
\text { exposure, report of } \\
\text { various symptoms } \\
\text { attributed to VDU } \\
\text { and/or powerlines, } \\
\text { abstinence } \\
\text { from smoking. }\end{array}$ & N.R/E. & Subjective report. \\
\hline $\begin{array}{l}\text { Regel et al., 2006 } \\
\text { (Switzerland) [50]. }\end{array}$ & Provocation & $\begin{array}{l}\text { Voluntary participation } \\
\text { after description } \\
\text { of the study in } \\
\text { advertisements in a } \\
\text { local newspaper, } \\
\text { flyers \& use of databases } \\
\text { of two previous studies } \\
\text { with IEI-EMF subjects } \\
\text { willing to participate in } \\
\text { future research projects. }\end{array}$ & $\begin{array}{l}\text { Base station- } \\
\text { specific }\end{array}$ & $\begin{array}{l}N=33, m . a=37.7 \\
f . g=57.5 \%\end{array}$ & $\begin{array}{l}\text { Self-reported } \\
\text { sensitivity to EMF } \\
\text { emitted by mobile } \\
\text { or cordless } \\
\text { phones \& antennas. }\end{array}$ & $\begin{array}{l}\text { Regular consumption } \\
\text { of narcotics or } \\
\text { psychoactive drugs } \\
\text { in the last } 6 \text { months, } \\
\text { smoking, diagnosed } \\
\text { with a chronic disease, } \\
\text { pregnancy, medical } \\
\text { history of head injuries, } \\
\text { neurologic/psychiatric } \\
\text { diseases, sleep } \\
\text { disturbances, } \\
\text { average alcohol } \\
\text { consumption of }>10 \\
\text { drinks per week, average } \\
\text { consumption of } \\
\text { caffeinated beverages } \\
\text { amounting to }>450 \\
\text { milligrams caffeine } \\
\text { per day, shift workers, } \\
\text { undertaking long-haul } \\
\text { flights of }>3 \text { hours time } \\
\text { zone difference within } \\
\text { the last month. }\end{array}$ & Subjective report. \\
\hline $\begin{array}{l}\text { Rubin et al., } 2006 \\
\text { (UK) [51]. }\end{array}$ & Provocation & $\begin{array}{l}\text { Through mailshots } \\
\text { organised by an } \\
\text { IEI-EMF support group, } \\
\text { advertisements \& articles } \\
\text { in health care institutions } \\
\text { \& practices. }\end{array}$ & MP-specific & $\begin{array}{l}N=71, m . a=37.1 \\
f . g=56 \%\end{array}$ & $\begin{array}{l}\text { Frequent experience } \\
\text { of headache-related } \\
\text { symptoms within } \\
20 \text { minutes of } \\
\text { using a } 900 \mathrm{MHz} \\
\text { GSM MP. }\end{array}$ & $\begin{array}{l}\text { Age of }<18 \text { or } \\
>75 \text { y.o, pregnancy, } \\
\text { psychotic illness, } \\
\text { use of antidepressants, } \\
\text { report of severe symptoms } \\
\text { at baseline while in } \\
\text { the testing room. }\end{array}$ & Subjective report. \\
\hline
\end{tabular}


Table 2 Experimental studies on IEI-EMF (Continued)

\begin{tabular}{|c|c|c|c|c|c|c|c|}
\hline $\begin{array}{l}\text { Eltiti et al., } 2007 \\
\text { (UK) [52]. }\end{array}$ & Provocation & $\begin{array}{l}\text { Voluntary participation } \\
\text { through local advertising, } \\
\text { IEI-EMF action groups } \\
\& \text { word of mouth. }\end{array}$ & $\begin{array}{l}\text { MP \& base } \\
\text { station-specific }\end{array}$ & $\begin{array}{l}N=56, m \cdot a=46.1 \\
f . g=42.9 \%\end{array}$ & $\begin{array}{l}\text { Individual experience } \\
\text { of negative health } \\
\text { effects attributed } \\
\text { to EMF emitted } \\
\text { from mobile } \\
\text { phone devices } \\
\text { and/or base } \\
\text { stations, based on } \\
\text { the "Electromagnetic } \\
\text { Hypersensitivity } \\
\text { Questionnaire" [53]. }\end{array}$ & $\begin{array}{l}\text { History of brain injury, } \\
\text { currently suffering from } \\
\text { epilepsy or claustrophobia, } \\
\text { undergone treatment for } \\
\text { mental disease or psycho- } \\
\text { active medication within } \\
4 \text { months before the study. }\end{array}$ & Subjective report. \\
\hline $\begin{array}{l}\text { Schröttner } \\
\text { et al., 2007 } \\
\text { (Austria) [54]. }\end{array}$ & Provocation & $\begin{array}{l}\text { Three different recruitment } \\
\text { sources: } \\
\text { 1. EMF self-help groups. } \\
\text { 2. Through advertisements } \\
\text { in local newspapers } \\
\text { \& inviting patients that } \\
\text { contacted a health care } \\
\text { service/institution for their } \\
\text { EMF-attributed symptoms. } \\
\text { 3. Subjects reporting severe } \\
\text { sleep problems being } \\
\text { deeply convinced that } \\
\text { these were caused by } \\
\text { EMF exposure. }\end{array}$ & General & $\begin{array}{l}\text { Recruitment } \\
\text { 1: } N=37, \\
\text { a.r }=27-81 \\
\text { f.g }=67.6 \% \\
\text { Recruitment } \\
\text { 2: } N=29 \\
\text { a.r }=32-63 \\
\text { f.g }=79 \% \\
\text { Recruitment } \\
\text { 3: } N=24 \\
\text { a.r }=37-73 \\
\text { f.g }=62.5 \% .\end{array}$ & $\begin{array}{l}\text { Self-reported } \\
\text { hypersensitivity } \\
\text { to electricity, } \\
\text { attribution of } \\
\text { symptoms to EMF, } \\
\text { active avoidance } \\
\text { behavior to } \\
\text { EMF sources. }\end{array}$ & $\begin{array}{l}\text { Sensitivity only to sources } \\
\text { of flickering light such as } \\
\text { VDU fluorescent tubes. }\end{array}$ & $\begin{array}{l}\text { Medical examination } \\
\text { (for part of the } \\
\text { group of } \\
\text { "Recruitment 2"). }\end{array}$ \\
\hline $\begin{array}{l}\text { Bamiou et al., } 2008 \\
\text { (UK) [55]. }\end{array}$ & Provocation & $\begin{array}{l}\text { Voluntary participation } \\
\text { after description of the } \\
\text { study through advertisements } \\
\text { at a health care services/ } \\
\text { institutions \& relative } \\
\text { website \& short film } \\
\text { shown on the national } \\
\text { television. }\end{array}$ & MP-specific & $\begin{array}{l}N=9, m \cdot a=36.7 \\
f . g=66.7\end{array}$ & $\begin{array}{l}\text { Report of headache } \\
\text { and/or disorientation, } \\
\text { dizziness, muzziness, } \\
\text { nausea attributed to } \\
\text { mobile telephone } \\
\text { use, age between } \\
20-55 \text { y.o, normal } \\
\text { tympanometry \& } \\
\text { normal pure } \\
\text { tone audiometric } \\
\text { thresholds } \\
\text { in both ears. }\end{array}$ & N.R/E. & $\begin{array}{l}\text { Subjective report, } \\
\text { audiometric } \\
\text { examination. }\end{array}$ \\
\hline $\begin{array}{l}\text { Hillert et al., } 2008 \\
\text { (Sweden) [56]. }\end{array}$ & Provocation & $\begin{array}{l}\text { Voluntary participation } \\
\text { after description of the } \\
\text { study in newspapers, } \\
\text { or individual initiative. }\end{array}$ & MP-specific & $\begin{array}{l}N=38, m \cdot a=28 \\
f . g=63.2 \%\end{array}$ & $\begin{array}{l}\text { Report of headache, } \\
\text { vertigo or } \\
\text { other kind of pain } \\
\text { or discomfort } \\
\text { in the head } \\
\text { attributed } \\
\text { to MP use. }\end{array}$ & $\begin{array}{l}\text { Attribution of symptoms } \\
\text { to sources other than MP, } \\
\text { medical or psychological } \\
\text { illness, undergoing } \\
\text { medication, sleep disorders, } \\
\text { hypertension, pregnancy, } \\
\text { history of severe injury. }\end{array}$ & Subjective report. \\
\hline
\end{tabular}


Table 2 Experimental studies on IEI-EMF (Continued)

\begin{tabular}{|c|c|c|c|c|c|c|c|}
\hline $\begin{array}{l}\text { Kwon et al., } 2008 \\
\text { (Finland) [57]. }\end{array}$ & Provocation & $\begin{array}{l}\text { Voluntary participation } \\
\text { after description of the } \\
\text { study in an } \\
\text { advertisement } \\
\text { that announced a } \\
\text { monetary prize. }\end{array}$ & MP-specific & $\begin{array}{l}\mathrm{N}=2 \text { male } \\
\text { subjects, } \\
\text { m.a }=37\end{array}$ & $\begin{array}{l}\text { Report of suffering } \\
\text { from severe } \\
\text { symptomatology } \\
\text { after use of a } \\
\text { mobile phone, high } \\
\text { score on a scale on } \\
\text { EMF sensibility } \\
\text { (defined as the } \\
\text { individual ability } \\
\text { to perceive EMF } \\
\text { without necessarily } \\
\text { developing symptoms). }\end{array}$ & $\begin{array}{l}\text { Neurological disease, } \\
\text { auditory abnormality, } \\
\text { being on permanent } \\
\text { medication. }\end{array}$ & Subjective report. \\
\hline $\begin{array}{l}\text { Landgrebe } \\
\text { et al., 2008a } \\
\text { (Austria \& } \\
\text { Germany) [58]. }\end{array}$ & Provocation & $\begin{array}{l}\text { Voluntary participation } \\
\text { after description of the } \\
\text { study in newspapers and } \\
\text { informative events at } \\
\text { public locations and } \\
\text { institutions. }\end{array}$ & General & $\begin{array}{l}N=88, m \cdot a=50.5 \\
f . g=58.4 \%\end{array}$ & $\begin{array}{l}\text { A symptom score of } \\
\text { at least } 19 \text { points on } \\
\text { the "Regensburger } \\
\text { EMF complaint list" } \\
\text { [59], attribution } \\
\text { of health symptoms } \\
\text { to named EMF sources } \\
\& \text { age between } \\
18-75 \text { y.o }\end{array}$ & $\begin{array}{l}\text { Unstable medical } \\
\text { condition. }\end{array}$ & Subjective report. \\
\hline $\begin{array}{l}\text { Leitgeb } \\
\text { et al., } 2008 \\
\text { (Austria \& } \\
\text { Germany) [60]. }\end{array}$ & $\begin{array}{l}\text { Crossover field } \\
\text { study }\end{array}$ & $\begin{array}{l}\text { Voluntary participation } \\
\text { after description of the } \\
\text { study in media. }\end{array}$ & General & $\begin{array}{l}N=43, m \cdot a=55.5 \\
f . g=60.5 \%\end{array}$ & $\begin{array}{l}\text { Personal conviction } \\
\text { on a causal role of } \\
\text { EMF indicated by the } \\
\text { employment of } \\
\text { precautionary activities } \\
\text { and/or measures (e.g. } \\
\text { reducing fields, } \\
\text { measuring exposure } \\
\text { in the household etc.), } \\
\text { above-normal } \\
\text { symptom scores on } \\
\text { standardized } \\
\text { questionnaires such } \\
\text { as the "Freiburger } \\
\text { Personality Inventory" } \\
\text { [61] and "PSQI" [62] } \\
\text { (at least } 5 \text { points } \\
\text { on the latter). }\end{array}$ & $\begin{array}{l}\text { Neurological \& } \\
\text { psychiatric } \\
\text { disorders, somatic } \\
\text { conditions that could } \\
\text { account for sleep } \\
\text { disturbances, drug } \\
\text { consumption less than } \\
2 \text { weeks before the study, } \\
\text { medical treatment for } \\
\text { severe conditions. }\end{array}$ & Subjective report. \\
\hline $\begin{array}{l}\text { Augner et al., } 2009 \\
\text { (Austria) [63]. }\end{array}$ & $\begin{array}{l}\text { Provocation } \\
\text { plus cross-sectional } \\
\text { data }\end{array}$ & Voluntary participation. & General & $\mathrm{N}=8, \mathrm{a} . \mathrm{r}=18-67$ & $\begin{array}{l}\text { Self-reported } \\
\text { electromagnetic } \\
\text { hypersensitivity } \\
\text { (rated as "strong" } \\
\text { or "very strong"). }\end{array}$ & N.R/E. & Subjective report. \\
\hline $\begin{array}{l}\text { Furubayashi } \\
\text { et al., 2009 } \\
\text { (Japan) [64]. }\end{array}$ & Provocation & $\begin{array}{l}\text { Randomly selected female } \\
\text { subjects }(\mathrm{N}=2472)\end{array}$ & $\begin{array}{l}\text { MP \& base } \\
\text { station-specific }\end{array}$ & $\mathrm{N}=11, \mathrm{~m} \cdot \mathrm{a}=37.3$ & $\begin{array}{l}\text { Report of symptoms } \\
\text { attributed to MP } \\
\text { use and/or exposure } \\
\text { to base stations, } \\
\text { symptoms should } \\
\text { persist "always" or } \\
\text { "almost always" }\end{array}$ & $\begin{array}{l}\text { History of myocardial } \\
\text { infarction epilepsy or } \\
\text { other (psycho) pathological } \\
\text { condition, undergoing } \\
\text { medical treatment for } \\
\text { severe medical conditions. }\end{array}$ & Subjective report. \\
\hline
\end{tabular}


Table 2 Experimental studies on IEI-EMF (Continued)

\begin{tabular}{|c|c|c|c|c|c|c|c|}
\hline $\begin{array}{l}\text { Nam et al., } 2009 \\
\text { (South Korea) [65]. }\end{array}$ & Provocation & $\begin{array}{l}\text { Voluntary participation } \\
\text { after description of the } \\
\text { study through } \\
\text { advertisements } \\
\text { at a health care } \\
\text { service/institution. }\end{array}$ & MP-specific. & $\begin{array}{l}N=18, m . a=26.1 \\
f . g=55.5 \%\end{array}$ & $\begin{array}{l}\text { Self-reported } \\
\text { hypersensitivity to } \\
\text { EMF emitted only } \\
\text { by CDMA } \\
\text { cellular phones. }\end{array}$ & $\begin{array}{l}\text { Self-reported } \\
\text { hypersensitivity } \\
\text { to other EMF sources, } \\
\text { subjects concerned } \\
\text { with payment for } \\
\text { volunteering, }\end{array}$ & Subjective report. \\
\hline $\begin{array}{l}\text { Szemerszky } \\
\text { et al., 2010 } \\
\text { (Hungary) [66]. }\end{array}$ & Provocation & $\begin{array}{l}\text { Voluntary participation } \\
\text { of university students. }\end{array}$ & General & N.R. & $\begin{array}{l}\text { Self-reported } \\
\text { electrosensitivity } \\
\text { (rated from "not } \\
\text { at all" to "fully"). }\end{array}$ & $\begin{array}{l}\text { Severe medical } \\
\text { disorders, health } \\
\text { conditions such as } \\
\text { premenstrual syndrome } \\
\text { and common cold that } \\
\text { could account for the } \\
\text { reported symptomatology }\end{array}$ & Subjective report. \\
\hline $\begin{array}{l}\text { Nieto-Hernandez } \\
\text { et al., } 2011 \\
\text { (UK) [67]. }\end{array}$ & Provocation & $\begin{array}{l}\text { Voluntary participation } \\
\text { after description of the } \\
\text { study within UK Police } \\
\text { Forces with the use of } \\
\text { circular emails, notices } \\
\text { in police newsletters } \\
\text { and intranet sites \& } \\
\text { advertisements in } \\
\text { police-related magazines } \\
\& \text { websites. }\end{array}$ & TETRA-specific & $\begin{array}{l}N=60, m . a=35.6, \\
f . g=11.7 \% .\end{array}$ & $\begin{array}{l}\text { Report of symptoms } \\
\text { attributed to } \\
\text { TETRA, report of } \\
\text { being at least } \\
70 \% \text { sure that the } \\
\text { radio signal } \\
\text { was the responsible } \\
\text { source, occurrence of } \\
\text { symptoms/sensations } \\
\text { within an hour of } \\
\text { radio use and } \\
\text { when the radio } \\
\text { was used } \\
\text { near the head. }\end{array}$ & $\begin{array}{l}\text { Pregnancy/trying to } \\
\text { conceive, medical or } \\
\text { psychological condition } \\
\text { which could account } \\
\text { for similar symptoms. }\end{array}$ & Subjective report. \\
\hline
\end{tabular}

Abbreviations: N.R., Not reported; N.R/E, Not reported or employed; EMF, Electromagnetic fields; IEI-EMF, Idiopathic environmental intolerance attributed to EMF; m.a, Mean age; a.r, Age range; f.g, Female gender distribution; y.o; Years old; MP, Mobile phone(s); VDT, Video display terminal: VDU, Video display units; GSM, Global system for mobile communications; CDMA, Code division multiple access; TETRA, Terrestrial trunked radio; PSQI, Pittsburgh sleep quality index. 
Table 3 Observational studies on IEI-EMF

\begin{tabular}{|c|c|c|c|c|c|c|c|}
\hline Reference (Country) & Study design & Recruitment & Type of sensitivity & $\begin{array}{l}\text { IEI-EMF sample } \\
\text { characteristics }\end{array}$ & $\begin{array}{l}\text { Identifying criteria } \\
\text { for IEI-EMF }\end{array}$ & $\begin{array}{l}\text { Main exclusion } \\
\text { criteria }\end{array}$ & $\begin{array}{l}\text { Identification/Case definition } \\
\text { methods for IEI-EMF }\end{array}$ \\
\hline $\begin{array}{l}\text { Bergdahl et al., } 1998 \\
\text { (Sweden) [68]. }\end{array}$ & Cross-sectional & $\begin{array}{l}\text { IEI-EMF subjects } \\
\text { referred to a } \\
\text { health care } \\
\text { service/institution. }\end{array}$ & $\begin{array}{l}\text { General, } \\
\text { VDU-specific }\end{array}$ & $\begin{array}{l}N=28 \\
m . a=45.5 \\
\text { f.g }=50 \% .\end{array}$ & $\begin{array}{l}\text { Report of } \\
\text { symptoms } \\
\text { assumed to } \\
\text { be caused by } \\
\text { VDU and/or } \\
\text { other EMF } \\
\text { sources. }\end{array}$ & N.R/E. & $\begin{array}{l}\text { Subjective report, } \\
\text { medical examination. }\end{array}$ \\
\hline $\begin{array}{l}\text { Hocking, } 1998 \\
\text { (Australia) [69]. }\end{array}$ & Cross-sectional & $\begin{array}{l}\text { Voluntary } \\
\text { participation } \\
\text { after description } \\
\text { of the study } \\
\text { in a medical } \\
\text { journal. }\end{array}$ & General & $\begin{array}{l}N=0 \\
\text { (people } \\
\text { identified } \\
\text { with IEI-EMF) }\end{array}$ & $\begin{array}{l}\text { Self-reported } \\
\text { electrosensitivity. }\end{array}$ & N.R/E. & Subjective report \\
\hline $\begin{array}{l}\text { Hillert et al., } 1999 \\
\text { (Sweden) [70]. }\end{array}$ & Case-control & $\begin{array}{l}\text { Subjects selected } \\
\text { from an older } \\
\text { occupational } \\
\text { health survey } \\
\& \text { IEl-EMF subjects } \\
\text { referred to a } \\
\text { health care } \\
\text { service/institution. }\end{array}$ & General & $\begin{array}{l}N=62, \\
\text { a.r }=20 \leq\end{array}$ & $\begin{array}{l}\text { Self-reported } \\
\text { hypersensitivity } \\
\text { to EMF. }\end{array}$ & N.R/E. & $\begin{array}{l}\text { Subjective report, } \\
\text { medical examination } \\
\text { (referred IEl-EMF } \\
\text { subjects). }\end{array}$ \\
\hline $\begin{array}{l}\text { Stockenius et al., } 2000 \\
\text { (Switzerland) [71]. }\end{array}$ & Cross-sectional & $\begin{array}{l}\text { Voluntary } \\
\text { participation of } \\
\text { male subjects } \\
\text { (mostly university } \\
\text { students) after } \\
\text { description of } \\
\text { the study } \\
\text { through } \\
\text { advertisements. }\end{array}$ & General & N.R. & $\begin{array}{l}\text { Self-reported } \\
\text { electrosensitivity } \\
\text { to named } \\
\text { sources } \\
\text { (ranked from } \\
\text { "very insensitive" } \\
\text { to "very } \\
\text { sensitive"). }\end{array}$ & N.R/E. & Subjective report. \\
\hline $\begin{array}{l}\text { Bergdahl et al., } 2001 \\
\text { (Sweden) [72]. }\end{array}$ & Cross-sectional & $\begin{array}{l}\text { IEI-EMF subjects } \\
\text { referred to a } \\
\text { health care } \\
\text { service/institution. }\end{array}$ & General & $\begin{array}{l}N=44 \\
\text { m.a }=47 \\
\text { f.g }=57 \%\end{array}$ & $\begin{array}{l}\text { Report of } \\
\text { symptoms } \\
\text { assumed to } \\
\text { be caused by } \\
\text { "abnormal } \\
\text { sensitivity } \\
\text { to EMF". }\end{array}$ & N.R/E. & $\begin{array}{l}\text { Subjective report, } \\
\text { medical interview } \\
\text { \& examination. }\end{array}$ \\
\hline $\begin{array}{l}\text { Hillert et al., } 2001 \\
\text { (Sweden) [73]. }\end{array}$ & Cross-sectional & $\begin{array}{l}\text { IEI-EMF subjects } \\
\text { referred to a } \\
\text { health care } \\
\text { service/institution. }\end{array}$ & General & $\begin{array}{l}N=14 \\
\text { m.a }=46 \\
\text { f.g }=64.3 \%\end{array}$ & $\begin{array}{l}\text { Self-reported } \\
\text { hypersensitivity } \\
\text { to EMF } \\
\text { including } \\
\text { disabling fatigue } \\
\text { attributed to } \\
\text { EMF exposure. }\end{array}$ & $\begin{array}{l}\text { Medical } \\
\text { condition } \\
\text { that could } \\
\text { account for } \\
\text { the reported } \\
\text { symptomatology. }\end{array}$ & $\begin{array}{l}\text { Subjective report, } \\
\text { medical examination. }\end{array}$ \\
\hline
\end{tabular}


Table 3 Observational studies on IEI-EMF (Continued)

\begin{tabular}{|c|c|c|c|c|c|c|c|}
\hline $\begin{array}{l}\text { Lyskov et al., } 2001 \\
\text { (Sweden) [74]. }\end{array}$ & Case-control & $\begin{array}{l}\text { IEI-EMF subjects } \\
\text { referred to a } \\
\text { health care } \\
\text { service/institution. }\end{array}$ & General & $\begin{array}{l}N=20 \\
m . a=47 \\
\text { f.g }=55 \%\end{array}$ & $\begin{array}{l}\text { Report of a } \\
\text { combined } \\
\text { pattern of } \\
\text { skin, general } \\
\text { and ocular } \\
\text { symptoms \& } \\
\text { attribution to } \\
\text { EMF exposure. }\end{array}$ & $\begin{array}{l}\text { Chronic } \\
\text { diseases, } \\
\text { acute illness } \\
\text { the last } 6 \\
\text { months, } \\
\text { undergoing } \\
\text { hormonal, } \\
\text { hypotensive } \\
\text { or sedative } \\
\text { therapy. }\end{array}$ & $\begin{array}{l}\text { Subjective report, } \\
\text { medical examination. }\end{array}$ \\
\hline $\begin{array}{l}\text { Hillert et al., } 2002 \\
\text { (Sweden) [6]. }\end{array}$ & Cross-sectional & $\begin{array}{l}\text { Randomly } \\
\text { selected sample } \\
\text { from general } \\
\text { population } \\
(\mathrm{N}=10605) \text {. }\end{array}$ & General & $\begin{array}{l}N=167 \\
a . r=19-80 \\
f . g=62.8 \%\end{array}$ & $\begin{array}{l}\text { Self-reported } \\
\text { hypersensitivity } \\
\text { to named } \\
\text { EMF sources. }\end{array}$ & N.R/E. & Subjective report. \\
\hline $\begin{array}{l}\text { Levallois et al., } \\
2002 \text { (USA) [8]. }\end{array}$ & Cross-sectional & $\begin{array}{l}\text { Randomly } \\
\text { selected sample } \\
\text { from general } \\
\text { population } \\
(\mathrm{N}=2072) \text {. }\end{array}$ & General & $\begin{array}{l}N=68 \\
\text { m.a }=43.4 \\
\text { f.g }=58.8 \%\end{array}$ & $\begin{array}{l}\text { Report of } \\
\text { being allergic } \\
\text { or very } \\
\text { sensitive } \\
\text { when being } \\
\text { near electrical } \\
\text { devices, } \\
\text { computers } \\
\text { and/or } \\
\text { powerlines. }\end{array}$ & N.R/E. & Subjective report \\
\hline $\begin{array}{l}\text { Stenberg et al., } \\
2002 \text { (Sweden) [75]. }\end{array}$ & Cross-sectional & $\begin{array}{l}\text { IEI-EMF subjects } \\
\text { referred to a } \\
\text { health care } \\
\text { service/institution. }\end{array}$ & $\begin{array}{l}\text { General, } \\
\text { VDT-specific. }\end{array}$ & $\begin{array}{l}\text { General } \\
\text { sensitivity: } \\
N=50 \\
\text { m.a }=49 \\
\text { f.g }=62 \% \\
\text { VDT specific: } \\
N=200 \\
\text { m.a }=50 \\
\text { f.g }=78.5 \% .\end{array}$ & $\begin{array}{l}\text { General: } \\
\text { Experience of } \\
\text { symptoms } \\
\text { attributed to } \\
\text { EMF sources in } \\
\text { general within } \\
24 \text { hours after } \\
\text { being exposed. } \\
\text { VDT-specific: } \\
\text { Experience of } \\
\text { (mainly skin) } \\
\text { symptoms } \\
\text { attributed to } \\
\text { VDT, TV screens } \\
\& \text { fluorescent } \\
\text { light within } \\
24 \text { hours after } \\
\text { being exposed. } \\
\text { For all subjects, } \\
\text { the possible } \\
\text { association } \\
\text { between exposure } \\
\& \text { symptoms } \\
\text { could not be } \\
\text { ruled out. }\end{array}$ & $\begin{array}{l}\text { Lack of } \\
\text { medical records } \\
\text { or examination, } \\
\text { diagnosed } \\
\text { medical } \\
\text { condition, } \\
\text { no symptom } \\
\text { attribution to } \\
\text { EMF within } \\
24 \text { hours } \\
\text { after being } \\
\text { exposed. }\end{array}$ & $\begin{array}{l}\text { Subjective report, } \\
\text { medical records } \\
\text { \& examination. }\end{array}$ \\
\hline
\end{tabular}


Table 3 Observational studies on IEI-EMF (Continued)

\begin{tabular}{|c|c|c|c|c|c|c|c|}
\hline $\begin{array}{l}\text { Sandström et al., } \\
2003 \text { (Sweden) [76]. }\end{array}$ & Case control & $\begin{array}{l}\text { IEI-EMF subjects } \\
\text { registered to a } \\
\text { health care } \\
\text { service/institution. }\end{array}$ & General & $\begin{array}{l}N=14 \\
m . a=48.9 \\
f . g=64.3 \%\end{array}$ & $\begin{array}{l}\text { Individual } \\
\text { perception that } \\
\text { exposure to VDT, } \\
\text { FTL, TV and/or } \\
\text { other EMF } \\
\text { sources causes } \\
\text { symptoms within } \\
24 \text { h, the possible } \\
\text { exposure-outcome } \\
\text { association could } \\
\text { not be ruled out. }\end{array}$ & $\begin{array}{l}\text { Symptoms } \\
\text { indicating } \\
\text { autonomic } \\
\text { nervous } \\
\text { dysregulation, } \\
\text { undergoing } \\
\text { hormonal or } \\
\text { hypotensive } \\
\text { therapy, } \\
\text { having } \\
\text { arrhythmia } \\
\text { due to } \\
\text { frequent } \\
\text { non-sinus } \\
\text { beats or } \\
\text { severe } \\
\text { cardiac } \\
\text { conduction } \\
\text { disturbances. }\end{array}$ & $\begin{array}{l}\text { Subjective report, } \\
\text { medical examination. }\end{array}$ \\
\hline $\begin{array}{l}\text { Bergdahl et al., } \\
2004 \text { (Sweden) [77]. }\end{array}$ & Case-control & $\begin{array}{l}\text { IEI-EMF subjects } \\
\text { referred and } \\
\text { registered to a } \\
\text { health care } \\
\text { service/institution. }\end{array}$ & General & $\begin{array}{l}N=250 \\
m . a=49.1 \\
f . g=75.2 \%\end{array}$ & $\begin{array}{l}\text { Individual } \\
\text { perception that } \\
\text { exposure to } \\
\text { VDT, TV and/or } \\
\text { other EMF } \\
\text { sources causes } \\
\text { symptoms within } \\
24 \mathrm{~h} \text {. }\end{array}$ & N.R/E. & $\begin{array}{l}\text { Subjective report, } \\
\text { medical examination. }\end{array}$ \\
\hline $\begin{array}{l}\text { Röösli et al., } 2004 \\
\text { (Switzerland) [4]. }\end{array}$ & Cross-sectional & $\begin{array}{l}\text { The survey was } \\
\text { described to } \\
\text { various local } \\
\text { institutions and } \\
\text { organizations } \\
\text { which informed } \\
\& \text { encouraged } \\
\text { IEl-EMF subjects } \\
\text { to participate. }\end{array}$ & General & $\begin{array}{l}N=394 \\
\text { m.a }=51 \\
\text { f.g }=57 \%\end{array}$ & $\begin{array}{l}\text { Report of } \\
\text { symptoms } \\
\text { (open question) } \\
\text { attributed to } \\
\text { EMF exposure. }\end{array}$ & N.R/E. & Subjective report. \\
\hline $\begin{array}{l}\text { Bergdahl et al., } 2005 \\
\text { (Sweden) [78]. }\end{array}$ & Case-control & $\begin{array}{l}\text { IEl-EMF subjects } \\
\text { referred to a } \\
\text { health care } \\
\text { service/institution. }\end{array}$ & General & $\begin{array}{l}N=33 \\
m . a=47 \\
f . g=51.5 \%\end{array}$ & $\begin{array}{l}\text { Report of } \\
\text { symptoms } \\
\text { assumed to be } \\
\text { caused by } \\
\text { sensitivity } \\
\text { to EMF. }\end{array}$ & N.R/E. & $\begin{array}{l}\text { Subjective report, } \\
\text { psychological examination. }\end{array}$ \\
\hline
\end{tabular}


Table 3 Observational studies on IEI-EMF (Continued)

\begin{tabular}{|c|c|c|c|c|c|c|c|}
\hline $\begin{array}{l}\text { * Carlsson et al., } 2005 \\
\text { (Sweden) [7]. }\end{array}$ & Cross-sectional & $\begin{array}{l}\text { Randomly } \\
\text { selected sample } \\
\text { from general } \\
\text { population } \\
\text { (N=13381), } \\
\text { based on } \\
\text { Östergren et al. } \\
\text { (report) [46]. }\end{array}$ & General & $\begin{array}{l}\mathrm{N}=2748 \\
\text { ("some } \\
\text { annoyance" } \\
\text { by EMF), } \\
\mathrm{N}=354 \\
\text { ("much } \\
\text { annoyance" } \\
\text { by EMF), } \\
\text { a.r }=18 \leq .\end{array}$ & $\begin{array}{l}\text { Individual } \\
\text { experience the } \\
\text { past } 2 \text { weeks } \\
\text { of "some" or } \\
\text { "much" } \\
\text { physiologic } \\
\text { "annoyance" } \\
\text { attributed to } \\
\text { FTL, and/or } \\
\text { VDU and/or } \\
\text { other electrical } \\
\text { equipment. }\end{array}$ & N.R/E. & Subjective report. \\
\hline $\begin{array}{l}\text { Eriksson et al., } 2006 \\
\text { (Sweden) [79]. }\end{array}$ & Cross-sectional & $\begin{array}{l}\text { Random sample } \\
\text { from general } \\
\text { population } \\
(\mathrm{N}=2154) \text {. }\end{array}$ & General & $\begin{array}{l}N=46 \\
\text { a.r }=18-64 \\
\text { f.g }=76 \%\end{array}$ & $\begin{array}{l}\text { For the past } 3 \\
\text { months, } \\
\text { report of } \\
5 \text { symptoms } \\
\text { on a weekly } \\
\text { basis and } 5 \\
\text { on a monthly } \\
\text { basis: These } \\
\text { symptoms could } \\
\text { be: fatigue, } \\
\text { feeling heavy- } \\
\text { headed, } \\
\text { headache, } \\
\text { concentration } \\
\text { difficulties, } \\
\text { itching, burning } \\
\text { or irritation } \\
\text { of the eyes, } \\
\text { dry eyes, dry } \\
\text { facial skin, } \\
\text { flushed facial } \\
\text { skin, itching/ } \\
\text { stinging/tight } \\
\text { or burning } \\
\text { sensation in } \\
\text { facial skin \& } \\
\text { cold hands } \\
\text { or feet. }\end{array}$ & N.R/E. & Subjective report. \\
\hline \multirow[t]{2}{*}{$\begin{array}{l}\text { Schreier et al., } 2006 \\
\text { (Switzerland) [10]. }\end{array}$} & Cross-sectional & $\begin{array}{l}\text { Randomly } \\
\text { selected sample } \\
\text { from general } \\
\text { population } \\
(\mathrm{N}=2048) \text {. }\end{array}$ & General & $\begin{array}{l}N=107 \\
\text { a.r }=14< \\
\text { f.g }=54.5 \%\end{array}$ & $\begin{array}{l}\text { Report of } \\
\text { adverse health } \\
\text { effects (open } \\
\text { question) } \\
\text { attributed to } \\
\text { EMF at the } \\
\text { time of the } \\
\text { interview or } \\
\text { anytime in } \\
\text { the past. }\end{array}$ & N.R/E. & Subjective report. \\
\hline & Cross-sectional & & General & & & N.R/E. & Subjective report. \\
\hline
\end{tabular}


Table 3 Observational studies on IEI-EMF (Continued)

\begin{tabular}{|c|c|c|c|c|c|c|c|}
\hline $\begin{array}{l}\text { Schüz et al., } 2006 \\
\text { (Germany) [80]. }\end{array}$ & & $\begin{array}{l}\text { Voluntary } \\
\text { participation } \\
\text { from EMF } \\
\text { self-help \& } \\
\text { action groups, } \\
\text { internet \& } \\
\text { media } \\
\text { advertisements, } \\
\text { invitation letters. }\end{array}$ & & $\begin{array}{l}N=107 \\
f . g=54 \%\end{array}$ & $\begin{array}{l}\text { Self-reported } \\
\text { hypersensitivity } \\
\text { to EMF. }\end{array}$ & & \\
\hline $\begin{array}{l}\text { Eltiti et al., } 2007 \\
\text { (UK) [53]. }\end{array}$ & $\begin{array}{l}\text { Three } \\
\text { cross-sectional } \\
\text { investigations. }\end{array}$ & $\begin{array}{l}\text { Investigation } \\
\text { 1\&3: IEI-EMF } \\
\text { subjects through } \\
\text { local self-help \& } \\
\text { action groups } \\
\text { or personal } \\
\text { contact. } \\
\text { Investigation } \\
\text { 2: Random selection } \\
\text { from the } \\
\text { general } \\
\text { population } \\
(\mathrm{N}=3633) \text {. }\end{array}$ & General & $\begin{array}{l}\text { Investigation } \\
1: N=50 \\
m . a=52.5 \\
\text { f.g }=66 \% \text {. } \\
\text { Investigation } \\
2: N=698 \text {. } \\
\text { Investigation } \\
3: N=88 \\
m . a=48.7 \\
\text { f.g }=53.4 \% .\end{array}$ & $\begin{array}{l}\text { Investigation } \\
\text { 1: Self-reported } \\
\text { sensitivity to } \\
\text { EMF, attribution } \\
\text { of symptoms } \\
\text { to EMF. } \\
\text { Investigation } \\
2 \& 3: \text { Self-reported } \\
\text { sensitivity to EMF. }\end{array}$ & N.R/E. & Subjective report. \\
\hline $\begin{array}{l}\text { Landgrebe et al., } 2007 \\
\text { (Germany) [81]. }\end{array}$ & Case-control & $\begin{array}{l}\text { Voluntary } \\
\text { participation } \\
\text { after description } \\
\text { of the study in } \\
\text { a local } \\
\text { newspaper. }\end{array}$ & General & $\begin{array}{l}N=23 \\
m . a=41.3 \\
f . g=74 \% .\end{array}$ & $\begin{array}{l}\text { Report of } \\
\text { severe } \\
\text { symptoms } \\
\text { that limited daily } \\
\text { functioning, } \\
\text { attribution of } \\
\text { these symptoms } \\
\text { to named EMF } \\
\text { sources \& age } \\
\text { between 18-64 y.o. }\end{array}$ & N.R/E. & Subjective report. \\
\hline $\begin{array}{l}\text { Hardell et al., } 2008 \\
\text { (Sweden) [82]. }\end{array}$ & Case-control & $\begin{array}{l}\text { Voluntary } \\
\text { participation. }\end{array}$ & General & $\begin{array}{l}\mathrm{N}=13 \\
\text { female } \\
\text { subjects, } \\
\mathrm{m} . \mathrm{a}=53 .\end{array}$ & $\begin{array}{l}\text { Report of symptoms } \\
\text { attributed to EMF. }\end{array}$ & $\begin{array}{l}\text { Severe medical } \\
\text { condition. }\end{array}$ & $\begin{array}{l}\text { Subjective report, } \\
\text { medical examination. }\end{array}$ \\
\hline $\begin{array}{l}\text { Lidmark et al., } 2008 \\
\text { (Sweden) [83]. }\end{array}$ & $\begin{array}{l}\text { Cross-sectional, } \\
\text { plus qualitative } \\
\text { data }\end{array}$ & $\begin{array}{l}\text { Voluntary } \\
\text { participation of } \\
\text { members of an } \\
\text { IEI-EMF self-help } \\
\text { group. }\end{array}$ & General & N.R. & $\begin{array}{l}\text { Report of symptoms } \\
\text { attributed to EMF }\end{array}$ & N.R/E. & $\begin{array}{l}\text { Subjective report, } \\
\text { medical \& psychiatric } \\
\text { examination. }\end{array}$ \\
\hline $\begin{array}{l}\text { Schröttner et al., } 2008 \\
\text { (Austria) [9]. }\end{array}$ & Cross-sectional & $\begin{array}{l}\text { Randomly } \\
\text { selected sample } \\
\text { from general } \\
\text { population } \\
(\mathrm{N}=526) \text {. }\end{array}$ & General & $\begin{array}{l}N=16 \\
a . r=15-80 \\
f . g=50 \%\end{array}$ & $\begin{array}{l}\text { Report of } \\
\text { disturbance/adverse } \\
\text { health effects (open } \\
\text { question) attributed } \\
\text { to named EMF } \\
\text { sources, looking for } \\
\text { medical help because } \\
\text { of symptom severity. }\end{array}$ & N.R/E. & Subjective report. \\
\hline
\end{tabular}


Table 3 Observational studies on IEI-EMF (Continued)

\begin{tabular}{|c|c|c|c|c|c|c|c|}
\hline $\begin{array}{l}\text { Dahmen et al., } 2009 \\
\text { (Germany) [84]. }\end{array}$ & Case-control & $\begin{array}{l}\text { Sample } \\
\text { selected from } \\
\text { EMF self-help } \\
\text { groups, an } \\
\text { internet-based } \\
\text { survey on EMF } \\
\text { and health \& } \\
\text { local } \\
\text { advertisements. }\end{array}$ & General & $\begin{array}{l}N=132 \\
m . a=51.5 \\
\text { f.g }=68.2 \%\end{array}$ & $\begin{array}{l}\text { A symptom } \\
\text { score } \\
\text { of at least } 14 \\
\text { points on the } \\
\text { "Regensburger } \\
\text { EMF complaint } \\
\text { list" [85], } \\
\text { attribution of } \\
\text { health symptoms } \\
\text { to named EMF } \\
\text { sources \& age } \\
\text { between } \\
\text { 18-56 y.o }\end{array}$ & $\begin{array}{l}\text { Acute } \\
\text { psychiatric } \\
\text { disorder (after } \\
\text { psychiatric } \\
\text { examination). }\end{array}$ & Subjective report. \\
\hline $\begin{array}{l}\text { Johansson et al., } 2010 \\
\text { (Sweden) [23]. }\end{array}$ & Case-control & $\begin{array}{l}\text { Voluntary } \\
\text { participation } \\
\text { after description } \\
\text { of the study in } \\
\text { newspaper } \\
\text { advertisements. }\end{array}$ & $\begin{array}{l}\text { General, } \\
\text { MP-specific, } \\
\text { VDT-specific }\end{array}$ & $\begin{array}{l}\text { MP-specific } \\
\text { sensitivity } \\
\text { group: } \\
N=45 \\
\text { m.a }=45.7 \\
\text { f.g }=62 \% \text {. } \\
\text { General } \\
\text { sensitivity } \\
\text { group: } \\
N=71 \\
\text { m.a }=51.6 \\
\text { f.g }=82 \% .\end{array}$ & $\begin{array}{l}\text { Report of } \\
\text { symptoms } \\
\text { attributed to: } \\
\text { 1. MP use only, } \\
\text { 2. VDT use only } \\
\text { or 3several types } \\
\text { of electrical } \\
\text { equipment. }\end{array}$ & N.R/E. & Subjective report. \\
\hline $\begin{array}{l}\text { Mohler et al., } 2010 \\
\text { (Switzerland) [86]. }\end{array}$ & Cross-sectional & $\begin{array}{l}\text { Randomly } \\
\text { selected sample } \\
\text { from general } \\
\text { population } \\
(\mathrm{N}=1212) \text {. }\end{array}$ & General & $N=253$. & $\begin{array}{l}\text { Self-reported } \\
\text { electrohypersensitivity } \\
\text { or report of } \\
\text { adverse health } \\
\text { effects } \\
\text { attributed } \\
\text { to EMF. }\end{array}$ & $\begin{array}{l}\text { Consumption of } \\
\text { sleeping pills, } \\
\text { night shift } \\
\text { workers, } \\
\text { severe disability. }\end{array}$ & Subjective report. \\
\hline $\begin{array}{l}\text { Nordin et al., } 2010 \\
\text { (Sweden) [87]. }\end{array}$ & Cross-sectional & $\begin{array}{l}\text { Voluntary } \\
\text { participation } \\
\text { of IEl subjects } \\
\text { after description } \\
\text { of the study } \\
\text { in a local } \\
\text { and a national } \\
\text { newspaper. }\end{array}$ & General & $\begin{array}{l}N=2, \\
a \cdot r=18-69 .\end{array}$ & $\begin{array}{l}\text { Report of } \\
\text { being } \\
\text { intolerant } \\
\text { to EMF. }\end{array}$ & Pregnancy. & Subjective report. \\
\hline $\begin{array}{l}\text { Röösli et al., } 2010 \\
\text { (Switzerland) [88]. }\end{array}$ & Cross-sectional & $\begin{array}{l}\text { Randomly } \\
\text { selected sample } \\
\text { from general } \\
\text { population } \\
(\mathrm{N}=1122) \text {. }\end{array}$ & General & $\begin{array}{l}N=130 \\
a . r=30-60 \\
f . g=72.3 \%\end{array}$ & $\begin{array}{l}\text { Self-reported } \\
\text { hypersensitivity } \\
\text { to EMF. }\end{array}$ & N.R/E. & Subjective report. \\
\hline
\end{tabular}

*Although the studies of Carlsson et al. [7] and Österberg et al. [45] are based on the same sample [46], they have some differences in terms of inclusion criteria and/or identification methods. This was the case also for the studies of Mohler et al. [86] and Röösli et al. [88].

Abbreviations: N.R., Not reported; N.R/E, Not reported or employed. FTL, Fluorescent tube light; EMF, Electromagnetic fields; IEI-EMF, Idiopathic environmental intolerance attributed to EMF; m.a, Mean age; a.r, Age range; f.g, Female gender distribution; y.o; Years old; MP, Mobile phone(s); VDT, Video display terminal: VDU, Video display units. 
relevant articles. A wide literature database held by the Mobile Phones Research Unit of King's College London was also consulted. A second literature search was carried out in order to update our review with studies published from May 2010 to June 2011.

\section{Inclusion criteria}

Only primary studies written in English and published in the peer-reviewed literature were considered as suitable for inclusion in the current review. Conference presentations, brief communications and reviews were excluded. The primary condition to include a study was the report of use of at least one criterion to identify individuals with IEI-EMF. Studies focusing on health effects from wider environmental exposures (such as chemicals) were eligible as long as they attempted to identify sensitivity to EMF in their investigation. Studies recruiting exclusively "healthy" individuals without any attempt to assess IEIEMF or identify relevant individuals were excluded. Since the "attribution" of health complaints to EMF is not necessarily synonymous with IEI-EMF and it is not an established prerequisite for its existence, studies relying solely on "attribution" without any mention of and explicit conceptual link with IEI-EMF or synonymous terms were not considered eligible for this review. Among papers based on the same sample and identifying criteria of IEI-EMF, the first publication was included.

\section{Data extraction}

For each included study, the following data were abstracted: reference and country, study design, methods and source of sample recruitment, IEI-EMF sample characteristics (such as sample size, age mean or range and gender distribution), type of sensitivity based on the triggering EMF source(s), the criteria used to identify individuals with IEI-EMF, exclusion criteria (based on selfreport/interview or clinical examination) and the case definition procedure followed for the identification of IEI-EMF (such as self-report and/or medical examination to exclude the possibility that a diagnosed disorder was responsible for the reported health complaints) (Tables 2 and 3). The data provided in the tables were derived from the information that was given or could be inferred from the original publications. However, in some cases (part of) the necessary information was not provided in the reviewed articles.

\section{Review Process}

The literature search was performed by the first author and the evaluation of inclusion criteria by $\mathrm{CB}, \mathrm{IVK}$ and GJR, with uncertainties resolved through consultation among all the authors. The initial screening was based on the titles and/or abstracts. Next, the hard copies of the potentially eligible publications were examined to assess whether they met the inclusion criteria.

\section{Results}

Search results

Figure 1 illustrates the literature search process. We examined 5328 citations in total and identified 35 experimental and 28 observational studies that met our inclusion criteria.

\section{Study characteristics}

When reported, sample sizes of subjects with IEI-EMF ranged between 1 to 100 in the experimental studies and from 2 to 2748 in the observational studies. The percentage of female participants (exempting case-studies) ranged between 0 to $81.3 \%$ and $50 \%$ to $100 \%$ respectively. In all studies, the reported mean age of IEIEMF individuals varied between 26.1 and 55.5 years. IEI-EMF triggered by several different EMF sources ("general") was the sensitivity type of primary focus in the included investigations $(\mathrm{n}=48)$, while 14 studies concentrated exclusively on "source-specific" IEI-EMF and three on both "general" and "source-specific" IEIEMF. There was a variety of synonyms of IEI-EMF in the literature such as "hypersensitivity (HS) to EMF", "electromagnetic Hypersensitivity (EHS)", "electrohypersensitivity", "environmental annoyance attributed to EMF", "electromagnetic distress syndrome" and "environmental illness". "Hypersensitivity to EMF" (and its variants) was by far the most frequently used definition/descriptive term (Figure 2). In 35 studies the case definition procedure was solely based on the subjective report of the respondents. In 28 studies it was mentioned that objective assessment (e.g. medical and/or psychological assessment) was additionally taken into account.

The principal method of sample recruitment was via study description in advertisements and/or local or national media (22 studies). The vast majority of the reviewed studies were conducted in Europe (58 studies).

\section{Experimental studies}

The major inclusion criteria used by experimental studies to identify individuals with IEI-EMF were:

- Attribution of NSPS to either various or specific sources of EMF (being reported 13 times).

- Self-reported IEI-EMF (or synonymous terms) $(\mathrm{n}=14)$.

- Experience of symptoms during or soon (from 20 minutes to 24 hours) after the individual perception or actual presence or use of an EMF exposure source $(\mathrm{n}=10)$.

- High score on a symptom scale $(n=6)$. 


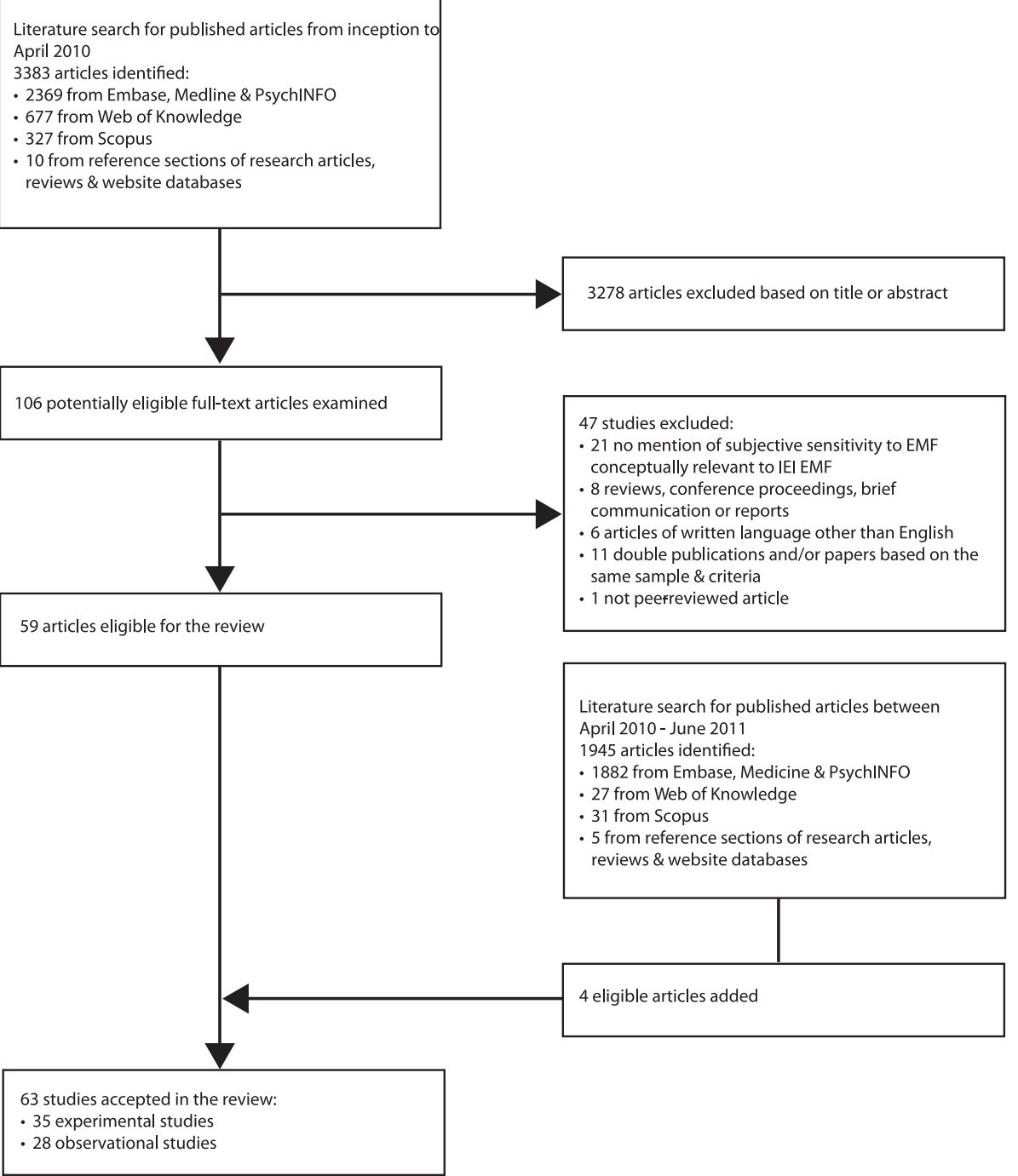

Figure 1 Flow diagram outlining the study selection process.

In addition, two studies used limitation in daily functioning of the individual due to the attributed health effects as an inclusion criterion.

The main exclusion criterion was the existence of a medical and/or psychiatric or psychological condition that could account for the reported health complaints $(n=20)$.

Other exclusion criteria included undergoing treatment for somatic or psychiatric conditions $(n=8)$, pregnancy $(n=5)$, history of severe injuries $(n=3)$ and regular smoking $(\mathrm{n}=2)$.

In 16 studies the case definition procedure did not only rely on subjective report, but also on medical and/ or psychiatric and/or psychological examination. In eight studies, the sample recruitment was based on participants who were already referred or registered to a health care institution (such as a university hospital) for their health complaints. All extracted data from the experimental studies are presented in Table 2.

\section{Observational Studies}

The major inclusion criteria used by observational studies to identify individuals with IEI-EMF were:

- Self-reported IEI-EMF (or synonymous terms) $(n=16)$.

- Attribution of NSPS to either various or specific EMF sources $(n=12)$.

- Experience of symptoms during or soon (from 20 minutes to 24 hours) after the individual perception or actual presence or use of an EMF exposure source $(n=3)$.

- Limitation in daily functioning of the individual due to the attributed health effects $(n=2)$. 


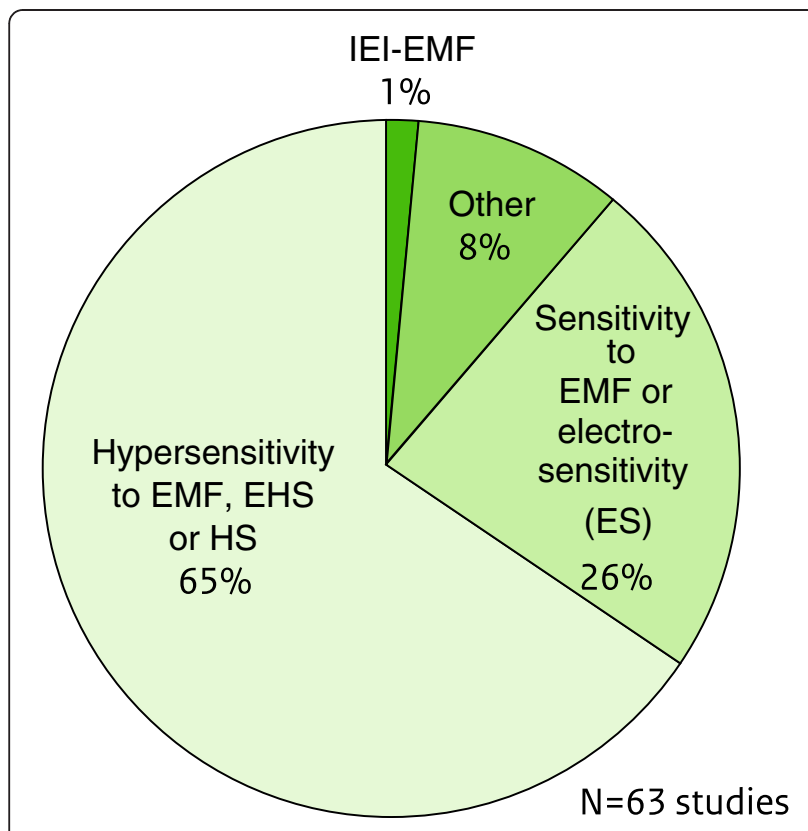

Figure 2 Distribution (\%) of terms used to describe IEI-EMF in the reviewed literature. Abbreviations: IEI-EMF, Idiopathic environmental intolerance attributed to EMF; EHS, Electrohypersensitivity; HS, Hypersensitivity.

The main exclusion criteria were a medical and/or psychiatric or psychological condition that could account for the reported health complaints and undergoing treatment for somatic or psychiatric condition $(n=4)$.

Eleven studies included medical and/or psychiatric and/or psychological examination to assess whether a pathological condition was responsible for patients' complaints. In nine studies the sample was based on participants who were already referred or registered to health care institutions for their complaints. All extracted data from the observational studies are listed in Table 3.

The prevalence of IEI-EMF in randomly selected samples of population-based epidemiological studies varied and seemed to be influenced by the number and degree of strictness of the applied identification criteria. This is illustrated in Figure 3. These differences could also be due to the population under study, year of study and sample stratification (e.g. age range).

\section{Discussion}

The present systematic review based on an extensive literature search, summarized the case definition criteria and methods that have been used in the published literature to date for the identification of subjects with IEIEMF.

It is noteworthy that only $1 \%$ of the reviewed studies used the term "IEI-EMF" as a descriptive term, despite the fact that it has been proposed by WHO since 2005 [5]. Sixty-five percent of the studies used the description "Hypersensitivity to EMF" which seems to be mainly characterized by the following aspects: Self-reported sensitivity to one or more sources of EMF, attribution of NSPS to either several or specific EMF sources (such as mobile phones and VDUs), experience of symptoms during or soon after (from 20 minutes to 24 hours) the individual perception or actual presence or use of an EMF source and absence of a (psycho)pathological condition accounting for the reported health complaints. In the majority of the studies the case definition procedure was based exclusively on self-report. In a smaller number of investigations, medical and/or psychiatric and/or psychological assessment was included.

In most of these studies participants were recruited from registries to a health care institution for their
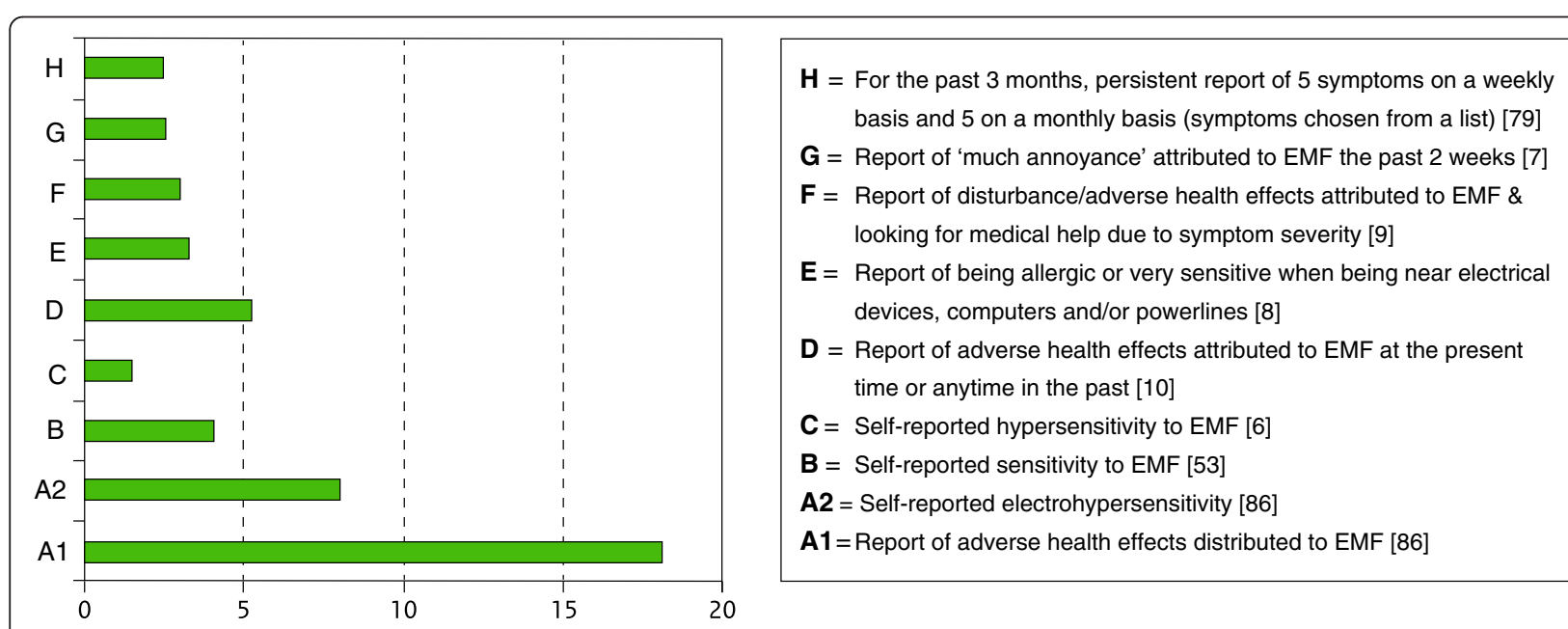

Figure 3 Prevalence (\%) of IEI-EMF based on the identifying criteria employed by population-based observational studies. 
symptoms and for whom medical data were available. Although there were no important differences between observational and experimental studies in the most frequently employed criteria, experimental studies used a larger number of criteria per investigation compared to observational studies. Moreover, the demographic profile of the recruited individuals with IEI-EMF in terms of age and gender was quite consistent; the frequency of female gender and age over 40 years were considerably higher in most of the studies.

Despite previous attempts to bring order to this field $[6,53,70]$, as it appears in the literature, IEI-EMF is still predominantly a self-reported sensitivity without a widely accepted and validated case definition tool. This could be due to the absence of a bioelectromagnetic mechanism [17] or because of the varying patterns regarding the symptom type, frequency and severity $[6,41]$. The other way around could also be the case: The lack of validated case definition criteria could have hindered the identification of homogeneous patient groups and consequently the recognition of symptom profiles and a physiologic mechanism. Furthermore, the application of very broad criteria could dilute the power of the studies and make difficult the detection of those individuals that really suffer from IEI-EMF. For example, although "Attribution" of NSPS to EMF could be considered as a first indication of suffering from IEIEMF, it is questionable whether it comprises a sufficient identifying criterion when used alone.

\section{Possible subgroups}

Several subdivisions may exist within IEI-EMF that may be of relevance to clinicians and researchers.

One such division is that between patients for whom an alternative diagnosis exists, which might account for their symptoms and those for whom it does not. The absence of screening for pathological conditions which might underlie the symptoms reported by participants in many studies was notable. Previous studies have identified occasionally high levels of other diagnoses in such patients, such as somatoform and anxiety disorders which might account for their ill-health $[89,90]$. Including these individuals in the same sample as those for whom there is no clear explanation for their symptoms may reduce our ability to identify causal factors for IEIEMF.

An additional distinction that we may need to take into account is between patients who attribute symptoms to short-term exposure to EMF and those for whom longer-term exposure is relevant. Furthermore, it remains unclear whether generalized and source-specific IEI-EMF should be assessed separately or not. Exposure from far-field sources such as high-voltage overhead powerlines and mobile phone base stations is mostly continuous and people often perceive it as less controllable compared to near-field sources such as mobile phones [10] but there is still no convincing evidence for source-specific sensitivities [13]. As differences may exist between IEI-EMF patients in terms of their psychological and health-related characteristics, division into subgroups for the purposes of research may be of use $[22,23]$. Perhaps the most complicated issue is to figure out whether self-reported-NSPS and objectively assessed physiologic reactions are preceded by events of the relevant (EMF) exposures, distinguishable from other random exposure events experienced during the day. Use of a prediction model based on modelled exposure from various sources [91,92] could be a solution; however it is questionable whether and how it could be systematically incorporated in a case definition tool.

Table 4 illustrates a number of proposed aspects for IEI-EMF, based on a synthesis of the existing identifying criteria in the reviewed literature. Considering the fact that the reported symptoms are quite common in the general population and also the lack of symptom patterns $[6,53]$ and etiology, the only parameter that clearly distinguishes sensitive from control individuals is the causal attribution of symptomatology to EMF exposure. Therefore, the attribution of health outcomes and selfreported sensitivity to EMF inevitably constitute, at the moment, the cornerstone of IEI-EMF case definition in research and clinical practice. Additional aspects such as medical examination/history would elucidate whether the reported health outcomes can be explained by underlying pathology. Cognitive and behavioral aspects could be complementarily included in the case definition, since evidence on their role in IEI-EMF is promising [18] but not yet established. Moreover, taking into account potentially harmful environmental agents other than EMF would be an important addition for research.

This is the first time that a systematic review is conducted on definitions and identifying criteria for IEIEMF. Given the large number of included articles, it is unlikely that any missing (or even excluded) studies would alter the results or increase any publication bias, especially since the aim of the current paper was not to assess etiologic associations.

It is a challenge how all the different case definition parameters for IEI-EMF can be concisely embodied in one international operational tool which could be used in research and clinical practice, and how this instrument could be adjusted to the possible cultural differences (e.g. in terms of wording/phrasing questions on health outcomes). Nevertheless, without the harmonization of the conceptual framework and validation of identifying criteria, the value of the case definition standards for IEI-EMF will remain insufficient and possibly unreliable. Apart from research, this has an important impact also in primary care; 
Table 4 Proposed case definition aspects for IEI-EMF

\begin{tabular}{|c|c|c|}
\hline \multirow[t]{2}{*}{ Dimensions of IEI-EMF } & \multicolumn{2}{|c|}{ Case definition assessment/identification of IEI-EMF } \\
\hline & Research & Clinical practice \\
\hline \multirow[t]{3}{*}{ Health effects } & - Subjective report of symptoms/physiologic reactions. & - Subjective report of symptoms/physiologic reactions. \\
\hline & $\begin{array}{l}\text { - The possibility that a known medical or psychiatric } \\
\text { condition is the cause of the reported health complaints } \\
\text { should be excluded with the use of standardized } \\
\text { interview and patient history. }\end{array}$ & $\begin{array}{l}\text { - The possibility that a known medical or psychiatric } \\
\text { condition is the cause of the reported health } \\
\text { complaints should be excluded after thorough } \\
\text { physical and psychiatric examination and } \\
\text { detailed patient history. }\end{array}$ \\
\hline & $\begin{array}{l}\text { - Current status of residential and occupational exposure } \\
\text { to harmful environmental agents that could be related } \\
\text { to the reported complaints (other than non-ionizing EMF). }\end{array}$ & \\
\hline \multirow[t]{2}{*}{ Triggering factors } & $\begin{array}{l}\text { - Attribution of NSPS or other physiologic reaction(s) } \\
\text { to either all/several EMF sources (General IEI-EMF) } \\
\text { or one specific EMF source (such as VDU, MP or } \\
\text { FTL)and/or }\end{array}$ & \\
\hline & $\begin{array}{l}\text { - Subjective report of being sensitive to specific or various } \\
\text { EMF sources. }\end{array}$ & \\
\hline \multirow[t]{2}{*}{ Cognition \& behavior (optional) } & $\begin{array}{l}\text { - Symptoms occur during or after the individual perception } \\
\text { or actual exposure, presence or use of an EMF source. }\end{array}$ & \\
\hline & $\begin{array}{l}\text { - Regular avoidance behavior towards EMF source(s) due to } \\
\text { the fear of a negative impact of EMF on health. }\end{array}$ & \\
\hline
\end{tabular}

physicians, who are often the first to be contacted by the sufferers, are usually not adequately informed about IEIEMF, which can affect the patient-doctor interaction and the management of the patient [26].

In order to properly construct an operational tool, a proposed two-phase approach can be briefly described as follows: In the first phase, a case definition and case selection tool should be developed, taking into account sources such as the published literature, expert opinions (e.g. based on a Delphi procedure [93]) and information on IEI-EMF patient characteristics from available datasets/ongoing research. At this stage, EMF measurements or provocation tests should not be a priority since a provocation study will only have added value after the formulation of a proper case definition and participant selection. Additionally, if the aim of a "case selection tool" is to routinely test cases where symptoms occur without a clear underlying pathology, then that tool should be concise, inexpensive and easy to implement, such as a short questionnaire or checklist. In the second phase, the case definition tool should be validated in terms of practical usability and the ability to differentiate between subgroups of IEI-EMF and patients with other conditions (e.g. chronic fatigue) who report similar symptoms. Based on the findings, the requirements for a follow up study could be outlined.

\section{Conclusions}

IEI-EMF is a poorly defined sensitivity. Heterogeneity and ambiguity of the existing definitions and criteria for IEI-EMF show the necessity to develop uniform criteria that will be applicable both in research and clinical practice. Broader criteria identified in the published literature such as attribution of NSPS to EMF and subjective report of being EMF sensitive could be used as a working definition for IEI-EMF which will serve as a basis for the development of a case selection tool. However, further optimization is required, testing its reliability and validity in several different patient groups, leading to an international multidisciplinary protocol with the active involvement of health care providers. This could also be a stepping stone for the harmonization of concepts and case definition for the broader condition of IEI.

\section{Abbreviations}

EMF: Electromagnetic Fields; EHS: Electrohypersensitivity; HS: Hypersensitivity; IEl: Idiopathic Environmental Intolerance; IEI-EMF: Idiopathic Environmental Intolerance attributed to Electromagnetic Fields; MP: Mobile Phone(s); NSPS: Non-Specific Physical Symptoms; VDU: Video Display Units; VDT: Video Display Terminals.

\section{Competing interests}

GJR has previously been funded by the UK Mobile Telecommunications and Health Research programme (www.MTHR.org.uk) and has acted as an expert witness for the Diocese of Norwich relating to the installation of Wifi on Church property. EL chairs the Science Forum of the Dutch Expertise Platform for Electromagnetic Fields (www.kennisplatform.nl/).

\section{Authors' contributions}

All the authors participate in the international multidisciplinary project INCORPORATE of the National Institute for Public Health and the Environment (RIVM). CB carried out the literature search, drafted the manuscript and incorporated input from all the rest authors in the manuscript. IVK and GJR conceived and coordinated the study and provided critical comments on the manuscript. EL provided critical comments on the manuscript. All authors have read and approved the final version of the manuscript. 


\section{Acknowledgements}

The current study is part of the project INCORPORATE "International collaboration on symptoms, psychological aspects and EMF exposure estimates: Towards harmonization and validation", funded by The Netherlands Organization for Health Research and Development (ZonMw). The study sponsor had no involvement in study design, writing and interpretation of the results and in the decision to submit the study for publication. The authors would like to thank Dr Rosa Nieto-Hernandez for her help in structuring the literature search protocol and Dr Rik Bogers for his feedback on the paper. In addition, the authors would like to thank Dr Joris Yzermans and Dr Mariette Hooiveld for their ideas regarding the suggestions for the development of a screening tool for IEI-EMF.

\section{Author details}

'Institute for Risk Assessment Sciences, Utrecht University, Utrecht, The Netherlands. ${ }^{2}$ National Institute for Public Health and the Environment (RIVM), Bilthoven, The Netherlands. ${ }^{3}$ King's College London, Department of Psychological Medicine, Institute of Psychiatry, London, UK.

\section{Received: 1 December 2011 Accepted: 7 August 2012}

Published: 11 August 2012

\section{References}

1. Randolph TG: Human ecology and susceptibility to the chemical environment. Springfield (IL): Charles Thomas; 1962.

2. Das-Munshi J, Rubin GJ, Wessely S: Multiple chemical sensitivities: A systematic review of provocation studies. J Allergy Clin Immunol 2006, 118:1257-1264.

3. Reed-Gibson P, Elms ANM, Ruding LA: Perceived Treatment Efficacy for Conventional and Alternative Therapies Reported by Persons with Multiple Chemical Sensitivity. Environ Health Perspect 2003, 111:1498-1504.

4. Röösli M, Moser M, Baldinini Y, Meier M, Braun-Fahrländer C: Symptoms of ill health ascribed to electromagnetic field exposure-A questionnaire survey. Int J Hygiene Environ Health 2004, 207:141-150.

5. WHO: Fact Sheet No. 296: Electromagnetic fields and public health. World Health Organization; 2005. http://www.emfandhealth.com/ WHO_EMSensitivity.pdf.

6. Hillert $L$, Berglind N, Arnetz BB, Bellander T: Prevalence of self-reported hypersensitivity to electric or magnetic fields in a population-based questionnaire survey. Scand J Work Environ Health 2002, 28:33-41.

7. Carlsson F, Karlson B, Orbaek P, Österberg K, Östergren PO: Prevalence of annoyance attributed to electrical equipment and smells in a Swedish population, and relationship with subjective health and daily functioning. Public Health 2005, 119x:568-577.

8. Levallois $P$, Neutra R, Lee G, Hristova L: Study of self-reported hypersensitivity to electromagnetic fields in California. Environ Health Perspect 2002, 110:619-623.

9. Schröttner J, Leitgeb N: Sensitivity to electricity - temporal changes in Austria. BMC Publ Health 2008, 8:310.

10. Schreier N, Huss A, Röösli M: The prevalence of symptoms attributed to electromagnetic field exposure: a cross-sectional representative survey in Switzerland. Soz Praventivmed 2006, 51:202-209.

11. Tseng MM, Lin YP, Cheng TJ: Prevalence and Psychiatric Co-Morbidity of Self-Reported Electromagnetic Field Sensitivity in Taiwan: A PopulationBased Study. Epidemiology 2008, 19:s108-s109.

12. Levallois $P$ : Hypersensitivity of human subjects to environmental electric and magnetic field exposure: A review of the literature. Environ Health Perspect 2002, 110:613-618.

13. Rubin GJ, Das-Munshi J, Wessely S: Electromagnetic hypersensitivity: A systematic review of provocation studies. Psychosom Med 2005, 67:224-232.

14. Röösli M: Radiofrequency electromagnetic field exposure and nonspecific symptoms of ill health: a systematic review. Environ Res 2008, 107:277-287.

15. Rubin GJ, Nieto-Hernandez R, Wessely S: Idiopathic environmental intolerance attributed to electromagnetic fields (formerly 'electromagnetic hypersensitivity'): An updated systematic review of provocation studies. Bioelectromagnetics 2009, 31:1-11.

16. Röösli M, Frei P, Mohler E, Hug K: Systematic review on the health effects of exposure to radiofrequency electromagnetic fields from mobile phone base stations. Bull World Health Organ 2010, 88:887-896.
17. Rubin GJ, Hillert L, Nieto-Hernandez R, Van Rongen E, Oftedal G: Do people with idiopathic environmental intolerance attributed to electromagnetic fields display physiological effects when exposed to electromagnetic fields? A systematic review of provocation studies. Bioelectromagnetics 2011, 32:593-609.

18. Rubin GJ: Das Munshi J, Wessely S: A systematic review of treatments for electromagnetic hypersensitivity. Psychother Psychosom 2006, 75:12-18.

19. Österberg K, Persson R, Karlson B, Carlsson EF, Orbaek P: Personality, mental distress, and subjective health complaints among persons with environmental annoyance. Hum Exp Toxicol 2007, 26:231-241.

20. Landgrebe M, Frick U, Hauser S, Langguth B, Rosner R, Hajak G, Eichhammer $P:$ Cognitive and neurobiological alterations in electromagnetic hypersensitive patients: Results of a case-control study. Psychol Med 2008, 38:1781-1791.

21. Persson R, Carlsson EF, Österberg K, Orbaek P, Karlson B: A two-week monitoring of self-reported arousal, worry and attribution among persons with annoyance attributed to electrical equipment and smells. Scand J Psychol 2008, 49:345-356.

22. Rubin GJ, Cleare AJ, Wessely S: Psychological factors associated with selfreported sensitivity to mobile phones. J Psychosom Res 2008, 64:1-9.

23. Johansson A, Nordin S, Heiden M, Sandström M: Symptoms, personality traits, and stress in people with mobile phone-related symptoms and electromagnetic hypersensitivity. J Psychosom Res 2010, 68:37-45.

24. Baliatsas C, Van Kamp I, Kelfkens G, Schipper M, Bolte J, Yzermans J, Lebret E: Non-specific physical symptoms in relation to actual and perceived proximity to mobile phone base stations and powerlines. BMC Publ Health 2011, 11:421.

25. Huss A, Röösli M: Consultations in primary care for symptoms attributed to electromagnetic fields - a survey among general practitioners. BMC Publ Health 2006, 6:267.

26. Berg-Beckhoff G, Heyer K, Kowall B, Breckenkamp J, Razum O: The views of primary care physicians on health risks from electromagnetic fields. Dtsch Arztebl Int 2010, 107:817-823.

27. Salomon D: Medical practitioners and electromagnetic fields (EMF): Testing their concern. Comptes Rendus Physique 2010, 11:636-640.

28. Rea WJ, Pan Y, Fenyves EJ, Sujisawa I, Samadi N, Ross GH: Electromagnetic field sensitivity. J Bioelectricity 1991, 10:241-256.

29. Hamnerius Y, Agrup G, Galt S, Nilsson R, Sandblom J, Lindgren R: Doubleblind provocation study of hypersensitivity reactions associated with exposure from VDUs. Preliminary short version. R Swed Acad Sci Rep 1993, 2:67-72.

30. Arnetz BB, Berg M, Anderzen I, Lundeberg T, Haker E: A nonconventional approach to the treatment of 'environmental illness'. J Occup Environ Med 1995, 37:838-844.

31. Andersson B, Berg M, Arnetz BB, Melin L, Langlet I, Liden SA: Cognitivebehavioral treatment of patients suffering from 'electric hypersensitivity': subjective effects and reactions in a double-blind provocation study. $J$ Occup Environ Med 1996, 38:752-758.

32. Bertoft G: Patient reactions to some electromagnetic fields from dental chair and unit: a pilot study. Swed Dent J 1996, 20:107-112.

33. Toomingas A: Provocation of the electromagnetic distress syndrome. Scand J Work Environ Health 1997, 22:457-458.

34. Sandström M, Lyskov E, Berglund A, Medvedev S, Hansson-Mild K: Neurophysiological Effects of Flickering Light in Patients with Perceived Electrical Hypersensitivity. J Occup Environ Med 1997, 39:15-22.

35. Hillert L, Hedman BK, Dolling BF, Arnetz BB: Cognitive behavioural therapy for patients with electric sensitivity - A multidisciplinary approach in a controlled study. Psychother Psychosom 1998, 67:302-310.

36. Trimmel M, Schweiger E: Effects of an ELF $(50 \mathrm{~Hz}, 1 \mathrm{mT})$ electromagnetic field (EMF) on concentration in visual attention, perception and memory including effects of EMF sensitivity. Toxicol Lett 1998, 96-97:377-382.

37. Flodin U, Seneby A, Tegenfeldt C: Provocation of electric hypersensitivity under everyday conditions. Scand J Work Environ Health 2000, 26:93-98.

38. Lonne-Rahm S, Andersson B, Melin L, Schultzberg M, Arnetz B, Berg M: Provocation with stress and electricity of patients with 'sensitivity to electricity. J Occup Environ Med 2000, 42:512-516.

39. Hillert L, Kolmodin-Hedman B, Eneroth P, Arnetz BB: The effect of supplementary antioxidant therapy in patients who report hypersensitivity to electricity: a randomized controlled trial. Medscape General Medicine 2001, 3:11. 
40. Lyskov E, Sandström M, Hansson-Mild K: Provocation study of persons with perceived electrical hypersensitivity and controls using magnetic field exposure and recording of electrophysiological characteristics. Bioelectromagnetics 2001, 22:457-462.

41. Hietanen M, Hamalainen AM, Husman T: Hypersensitivity symptoms associated with exposure to cellular telephones: no causal link. Bioelectromagnetics 2002, 23:264-270.

42. Hillert L, Savlin P, Levy BA, Heidenberg A, Kolmodin-Hedman B: Environmental illness - Effectiveness of a salutogenic group-intervention programme. Scand J Public Health 2002, 30:166-175.

43. Mueller $\mathrm{CH}$, Krueger $\mathrm{H}$, Schierz $\mathrm{C}$ : Project NEMESIS: perception of a $50 \mathrm{~Hz}$ electric and magnetic field at low intensities (laboratory experiment). Bioelectromagnetics 2002, 23:26-36.

44. Leitgeb N, Schröttner J: Electrosensibility and electromagnetic hypersensitivity. Bioelectromagnetics 2003, 24:387-394.

45. Österberg K, Persson R, Karlson B, Orbæk P: Annoyance and performance in three groups of environmentally intolerant subjects during experimental challenge with chemical odors. Scand J Work Environ Health 2004, 6:486-496.

46. Ostergren PO, Merlo J, Lindström M, Rosvall M, Kahn FA, Lithman T: Hälsoförhållanden i Skåne. Folkhälsoenkät Skåne 2000 (Report in Swedish). Region Skåne: Kommunförbundet Skåne och Skåne läns allmänna Försäkringskassa; 2001.

47. Belyaev IY, Hillert L, Protopopova M, Tamm C, Malmgren LOG, Persson BRR, Selivanova G, Harms-Ringdahl M: $915 \mathrm{MHz}$ microwaves and $50 \mathrm{~Hz}$ magnetic field affect chromatin conformation and 53BP1 foci in human lymphocytes from hypersensitive and healthy persons. Bioelectromagnetics 2005, 26:173-184.

48. Frick U, Kharraz A, Hauser S, Wiegand R, Rehm J, Von Kovatsits U, Eichhammer P: Comparison perception of singular transcranial magnetic stimuli by subjectively electrosensitive subjects and general population controls. Bioelectromagnetics 2005, 26:287-298.

49. Wenzel F, Reissenweber J, David E: Cutaneous microcirculation is not altered by a weak $50 \mathrm{~Hz}$ magnetic field. Biomed Tech 2005, 50:14-18.

50. Regel SJ, Negovetic S, Röösli M, Berdinas V, Schuderer J, Huss A, Lott U, Kuster N, Achermann P: UMTS base stationlike exposure, well-being, and cognitive performance. Environ Health Perspect 2006, 114:1270-1275.

51. Rubin GJ, Hahn G, Everitt B, Cleare AJ, Wessely S: Are some people sensitive to mobile phone signals? A within participants, double-blind, randomised provocation study. Br Med J 2006, 332:886-889.

52. Eltiti S, Wallace D, Ridgewell A, Zougkou K, Russo R, Sepulveda F, MirshekarSyahkal D, Rasor P, Deeble R, Fox E: Does short-term exposure to mobile phone base station signals increase symptoms in individuals who report sensitivity to electromagnetic fields? A double- blind randomized provocation study. Environ Health Perspect 2007, 115:1603-1608.

53. Eltiti S, Wallace D, Zougkou K, Russo R, Joseph S, Rasor P, Fox E: Development and evaluation of the electromagnetic hypersensitivity questionnaire. Bioelectromagnetics 2007, 28:137-151.

54. Schröttner J, Leitgeb N, Hillert L: Investigation of Electric Current Perception Thershold of Different EHS Groups. Bioelectromagnetics 2007, 28:208-213.

55. Bamiou DE, Ceranic B, Cox R, Watt H, Chadwick P, Luxon LM: Mobile telephone use effects on peripheral audiovestibular function: A case- control study. Bioelectromagnetics 2008, 29:108-117.

56. Hillert L, Akerstedt T, Lowden A, Wiholm C, Kuster N, Ebert S, Boutry C, Moffat SD, Berg M, Arnetz BB: The effects of $884 \mathrm{MHz}$ GSM wireless communication signals on headache and other symptoms: An experimental provocation study. Bioelectromagnetics 2008, 29:185-196.

57. Kwon MS, Koivisto M, Laine M, Hamalainen H: Perception of the electromagnetic field emitted by a mobile phone. Bioelectromagnetics 2008, 29:154-159.

58. Landgrebe M, Barta W, Rosengarth K, Frick U, Hauser S, Langguth B, Rutschmann R, Greenlee MW, Hajak G, Eichhammer P: Neuronal correlates of symptom formation in functional somatic syndromes: A fMRI study. Neurolmage 2008, 41:1336-1344.

59. Frick U, Mayer M, Hauser S, Binder H, Rosner R, Eichhammer P: Entwicklung eines deutschsprachigen Messinstruments für "ElektrosmogBeschwerden" (in German). Umweltmedizin in Forschung \& Praxis 2006, $11: 11-22$.

60. Leitgeb N, Schröttner J, Cech R, Kerbl R: EMF-protection sleep study near mobile phone base stations. Somnologie 2008, 12:234-243.
61. Fahrenberg J: Die Freiburger Beschwerdenliste (FBL) (in German). Z Klin Psychol 1975, 4:79-100.

62. Buysse DJ, Reynolds CF, Monk TH, Berman SR, Kupfer DJ: The Pittsburgh Sleep Quality Index: a new instrument for psychiatric practice and research. Psychiatry Res 1989, 28:193-213.

63. Augner C, Florian M, Pauser G, Oberfeld G, Hacker GW: GSM base stations: Short-term effects on well-being. Bioelectromagnetics 2009, 30:73-80.

64. Furubayashi T, Ushiyama A, Terao $Y$, Mizuno $Y$, Shirasawa $K$, Pongpaibool $P$, Simba AY, Wake K, Nishikawa M, Miyawaki K, Yasuda A, Uchiyama M, Yamashita HK, Masuda H, Hirota S, Takahashi M, Okano T, Inomata-Terada S, Sokejima S, Maruyama E, Watanabe S, Taki M, Ohkubo C, Ugawa Y: Effects of short-term W-CDMA mobile phone base station exposure on women with or without mobile phone related symptoms. Bioelectromagnetics 2009, 30:100-113.

65. Nam KC, Lee JH, Noh HW, Cha EJ, Kim NH, Kim DW: Hypersensitivity to RF fields emitted from CDMA cellular phones: A provocation study. Bioelectromagnetics 2009, 30:641-650.

66. Szemerszky R, Koteles F, Lihi R, Bardos G: Polluted places or polluted minds? An experimental sham-exposure study on background psychological factors of symptom formation in 'Idiophatic Environmental Intolerance attributed to electromagnetic fields'. Int J Hyg Environ Health 2010, 213:387-394

67. Nieto-Hernandez R, Williams J, Cleare AJ, Landau S, Wessely S, Rubin GJ: Can exposure to a terrestrial trunked radio (TETRA)-like signal cause symptoms? A randomized double-blind provocation study. Occup Environ Med 2011, 68:339-344.

68. Bergdahl J, Tillberg A, Stenman E: Odontologic survey of referred patients with symptoms allegedly caused by electricity or visual display units. Acta Odontol Scand 1998, 56:303-307.

69. Hocking B: Preliminary report: symptoms associated with mobile phone use. Occup Med 1998, 48:357-360.

70. Hillert L, Hedman BK, Söderman E, Arnetz BB: Hypersensitivity to electricity: Working definition and additional characterization of the syndrome. $J$ Psychosom Res 1999, 47:429-438.

71. Stockenius S, Brugger P: Perceived electrosensitivity and magical ideation. Percept Mot Skills 2000, 90:899-900.

72. Bergdahl J, Bergdahl M: Environmental illness: evaluation of salivary flow, symptoms, diseases, medications, and psychological factors. Acta Odontol Scand 2001, 59:104-110.

73. Hillert L, Flato S, Georgellis A, Arnetz BB, Kolmodin-Hedman B: Environmental illness: Fatigue and cholinesterase activity in patients reporting hypersensitivity to electricity. Environ Res 2001, 85:200-206.

74. Lyskov E, Sandström M: Hansson Mild K: Neurophysiological study of patients with perceived electrical hypersensitivity. Int J Psychophysiol 2001, 42:233-241.

75. Stenberg B, Bergdahl J, Edvardsson B, Eriksson N, Lindén G, Widman L: Medical and social prognosis for patients with perceived hypersensitivity to electricity and skin symptoms related to the use of visual display terminals. Scand J Work Environ Health 2002, 28:349-357.

76. Sandström M, Lyskov E, Hörnsten R: Hansson-Mild, Wiklund U, Rask P, Klucharev V, Stenberg B, Bjerle P: ECG monitoring in patients with perceived electrical hypersensitivity. Int J Psychophysiol 2003, 49:227-235.

77. Bergdahl J, Stenberg B, Eriksson N, Lindén G, Widman L: Coping and selfimage in patients with visual display terminal-related skin symptoms andperceived hypersensitivity to electricity. Int Arch Occup Environ Health 2004, 77:538-542.

78. Bergdahl J, Marell L, Bergdahl M, Perris H: Psychobiological personality dimensions in two environmental-illness patient groups. Clin Oral Investig 2005, 9:251-256

79. Eriksson NM, Stenberg BG: Baseline prevalence of symptoms related to indoor environment. Scand J Public Health 2006, 34:387-396.

80. Schüz J, Petters C, Egle UT, Jansen B, Kimbel R, Letzel S, Nix W, Schmidt LG, Vollrath L: The "Mainzer EMF-Wachhund": results from a watchdog project on self-reported health complaints attributed to exposure to electromagnetic fields. Bioelectromagnetics 2006, 27:280-287.

81. Landgrebe M, Hauser S, Langguth B, Frick U, Hajak G, Eichhammer P: Altered cortical excitability in subjectively electrosensitive patients: results of a pilot study. J Psychosom Res 2007, 62:283-288.

82. Hardell L, Carlberg M, Söderqvist F, Hardell K, Björnfoth H, van Bavel B, Lindström G: Increased concentrations of certain persistent organic 
pollutants in subjects with self-reported electromagnetic

hypersensitivity-a pilot study. Electromagn Biol Med 2008, 27:197-203.

83. Lidmark AM, Wikmans T: Are they really sick? A report on persons who are electrosensitive and/or injured by dental material in Sweden. Journal of Orthomolecular Medicine 2008, 23:153-160.

84. Dahmen N, Ghezel-Ahmadi D, Engel A: Blood Laboratory Findings in Patients Suffering From Self-Perceived Electromagnetic Hypersensitivity (EHS). Bioelectromagnetics 2009, 30:299-306.

85. Frick U, Rehm J, Eichhammer P: Risk perception, somatization, and self report of complaints related to electromagnetic fields-a randomized survey study. Int I Hyg Environ Health 2002, 205:353-360.

86. Mohler E, Frei P, Fahrländer CB, Fröhlich J, Neubauer G, Röösli M: Effects of Everyday Radiofrequency Electromagnetic-Field Exposure on Sleep Quality: A Cross-Sectional Study. Radiat Res 2010, 174:347-356.

87. Nordin M, Andersson L, Nordin S: Coping strategies, social support and responsibility in chemical Intolerance. J Clin Nurs 2010, 19:2162-2173.

88. Röösli M, Mohler E, Frei P: Sense and sensibility in the context of radiofrequency electromagnetic field exposure. Comptes Rendus Physique 2010, 11:576-584.

89. Bornschein S, Hausteiner C, Zilker T, Förstl H: Psychiatric and somatic disorders and multiple chemical sensitivity (MCS) in 264 environmental patients. Psychol Med 2002, 32:1387-1394.

90. Bailer J, Witthöft M, Paul C, Bayerl C, Rist F: Evidence for overlap between idiopathic environmental intolerance and somatoform disorders.

Psychosom Med 2005, 67:921-929.

91. Frei $P$, Mohler E, Bürgi A, Fröhlich J, Neubauer G, Braun-Fahrländer C, Röösli $\mathrm{M}: \mathrm{A}$ prediction model for personal radio frequency electromagnetic field exposure. Sc of the Tot Env. 2009, 408:102-108.

92. Frei $P$, Mohler E, Bürgi A, Fröhlich J, Neubauer G, Braun-Fahrländer C, Röösli M: Classification of personal exposure to radio frequency electromagnetic fields (RF-EMF) for epidemiological research: Evaluation of different exposure assessment methods. Environ Int 2010, 36:714-720.

93. Hasson F, Keeney S, McKenna H: Research quidelines for the Delphi survey technique. J Adv Nurs 2000, 32:1008-1015.

doi:10.1186/1471-2458-12-643

Cite this article as: Baliatsas et al:: Idiopathic environmental intolerance attributed to electromagnetic fields (IEI-EMF): A systematic review of identifying criteria. BMC Public Health 2012 12:643.

\section{Submit your next manuscript to BioMed Central and take full advantage of:}

- Convenient online submission

- Thorough peer review

- No space constraints or color figure charges

- Immediate publication on acceptance

- Inclusion in PubMed, CAS, Scopus and Google Scholar

- Research which is freely available for redistribution 